\title{
Plant and vegetation functional responses to cumulative high nitrogen deposition in rear-edge heathlands
}

\author{
Angela Taboada*, Javier Calvo-Fernández, Elena Marcos, Leonor Calvo \\ Area of Ecology, University of León, E-24071 León, Spain \\ Institute of Environmental Research (IMA), University of Léon, E-24071 León, Spain
}

\section{A R T I C L E I N F O}

\section{Article history:}

Received 6 March 2018

Received in revised form 7 May 2018

Accepted 7 May 2018

Available online $\mathrm{xxx}$

Editor: Elena PAOLETTI

\section{Keywords:}

Air pollution

Calluna vulgaris heathlands

Cumulative nitrogen effect

Life cycle stage

Nitrogen saturation

Rear-edge populations

\begin{abstract}
A B S T R A C T
Elevated atmospheric nitrogen $(\mathrm{N})$ deposition is a major driver of change, altering the structure/functioning of nutrient-poor Calluna vulgaris-heathlands over Europe. These effects amply proven for north-western/ central heathlands may, however, vary across the ecosystem's distribution, especially at the range limits, as heathlands are highly vulnerable to land-use changes combined with present climate change. This is an often overlooked and greatly understudied aspect of the ecology of heathlands facing global change. We investigated the effects of five $\mathrm{N}$-fertilisation treatments simulating a range of $\mathrm{N}$ deposition rates $(0,10,20$, and $50 \mathrm{~kg} \mathrm{Nha}^{-1} \mathrm{yr}^{-1}$ for 1 year; and $56 \mathrm{~kg} \mathrm{Nha}^{-1} \mathrm{yr}^{-1}$ for 9 years) on the Calluna-plants, the plant functional groups, species composition and richness of two life-cycle stages (building/young- and mature-phase) of Calluna-heathlands at their rear-edge limit. Our findings revealed a dose-related response of the shoot length and number of flowers of young and mature Calluna-plants to the addition of N, adhering to the findings from other heathland locations. However, cumulative high-N loading reduced the annual growth and flowering of young plants, showing early signs of $\mathrm{N}$ saturation. The different plant functional groups showed contrasting responses to the cumulative addition of $\mathrm{N}$ : annual/perennial forbs and annual graminoids increased with quite low values; perennial graminoids were rather abundant in young heathlands but only slightly augmented in mature ones; while bryophytes and lichens strongly declined at the two heathland life-cycle stages. Meanwhile there were no significant N-driven changes in plant species composition and richness. Our results demonstrated that Calluna-heathlands at their low-latitude distribution limit are moderately resistant to cumulative high-N loading. As north-western/central European heathlands under high-N inputs broadly experienced the loss of plant diversity and pronounced changes in plant species dominance, rear-edge locations may be of critical importance to unravel the mechanisms of heathland resilience to future global change.
\end{abstract}

(C) 2017

\section{Introduction}

Human activities have exceedingly increased the mobility and deposition of biologically reactive forms of nitrogen $(\mathrm{N})$ in recent decades worldwide (Galloway et al., 2004, 2008; Peñuelas et al., 2017). This anthropogenic disruption of the global $\mathrm{N}$ cycle is responsible for biodiversity loss and altered ecosystem processes in a great variety of terrestrial ecosystems (e.g., Bobbink et al., 2010; Ochoa-Hueso et al., 2011). What is more, cumulative $\mathrm{N}$ inputs are one of the most significant factors producing unprecedented changes in the functioning of ecosystems, even at low deposition rates (Clark and Tilman, 2008; De Schrijver et al., 2011; Humbert et al., 2016; Phoenix et al., 2012). Understanding the magnitude of the effects of long-term airborne $\mathrm{N}$ depositions is, therefore, of major importance for the preservation of ecosystems, in particular, semi-natural habitats

* Corresponding author at: Area of Ecology, Department of Biodiversity and Environmental Management, University of León, Campus Vegazana s/n, E-24071 León, Spain.

Email address: angela.taboada@unileon.es (A. Taboada) adapted to low levels of $\mathrm{N}$ availability (Field et al., 2014; Humbert et al., 2016; Phoenix et al., 2012; Soons et al., 2017; Stevens et al., 2016).

Heathlands dominated by the dwarf shrub Calluna vulgaris (L.) Hull (henceforth referred to as Calluna) are semi-natural ecosystems on nutrient-poor soils pertaining to traditional cultural landscapes within Atlantic Europe (Loidi et al., 2010), highly endangered across their entire distribution range (Fagúndez, 2013). Land-use changes (abandonment or intensification), $\mathrm{N}$ pollution, natural succession, and invasion by exotic species are the main drivers causing heathland habitat destruction and fragmentation throughout Europe (e.g., Bartolomé et al., 2005; Britton et al., 2017; Henning et al., 2017; Rose et al., 2000). Many heathland field-surveys and N-manipulation experiments over different temporal scales have strongly demonstrated that elevated $\mathrm{N}$ deposition leads to (1) changes in plant growth, phenology and chemistry (e.g., Bähring et al., 2017; Britton and Fisher, 2008), (2) increased plant sensitivity to abiotic/biotic stressors (e.g., Heil and Diemont, 1983; Prins et al., 1991), (3) a reduction in plant diversity (Britton and Fisher, 2007; Maskell et al., 2010; Southon et al., 2013), (4) the loss of N-sensitive plant species 
(Caporn et al., 2014; Van den Berg et al., 2008), and (5) the invasion by nitrophilous species (Britton and Fisher, 2007; Southon et al., 2013). Moreover, a few long-term researches demonstrated the lasting nature of the majority of these $\mathrm{N}$-driven impacts on the heathland ecosystem (Carroll et al., 1999; Field et al., 2017; Power et al., 2006; Southon et al., 2012) that are expected to continue over coming decades (Payne et al., 2017; Stevens et al., 2016). These studies, however, have principally been accomplished in north-western and central European Calluna-heathlands; very little is known about the responses of southern heathlands at the rear-edge of the ecosystem's distribution to elevated $\mathrm{N}$ availability (Calvo et al., 2005, 2007; Cuesta et al., 2008; Marcos et al., 2003), especially over the long-term (Calvo-Fernández et al., 2018; Taboada et al., 2016). This lack of knowledge impedes the implementation of proper on-site management to counterbalance the potentially detrimental impacts of $\mathrm{N}$ loading in rear-edge heathland locations that are also vulnerable to present climate change (Fagúndez, 2013). Whereas for other heathland areas across Europe, diverse management measures (e.g., grazing, mowing, prescribed burning, sod-cutting; Härdtle et al., 2009; Von Oheimb et al., 2009) have been tested for their effectiveness in maintaining the low-nutrient status of the given heathland location with minor adverse impacts on the ecosystem (see review by Jones et al., 2017).

Recent researches have shown that environmental factors affect species differentially at the centre and the edges of their distribution ranges (e.g., Pearson et al., 2009; Viejo et al., 2011; see review by Sagarin et al., 2006), with notable dissimilarities between leading and rear edges (i.e., high- and low-latitude limits, respectively; Hampe and Petit, 2005). This generally implies that findings from a given location within a species' range might not be applicable to other parts of the range (Sagarin et al., 2006). Within the broad distribution area of $\mathrm{Cal}$ luna-heathlands across Europe, it is very likely that dominant Calluna plants from different locations have evolved particular adaptive traits to their local conditions (Loidi et al., 2010) and are, thus, differentially sensitive to environmental changes. To our knowledge, though, only one study (Meyer-Grünefeldt et al., 2016) has experimentally assessed how central and marginal Calluna plants responded to global change testing the single and combined effects of drought and $\mathrm{N}$ deposition. This study concluded that (1-2-year-old) plants from the southern-most limit of the Calluna-heathland ecosystem's distribution are better adapted to drought events than those from the centre of the range, except under elevated $\mathrm{N}$ availability. It is, however, difficult to extrapolate the results of these short-term effects on potted Calluna seedlings to long-term effects on more complex natural systems. Further research is, therefore, necessary to investigate the differential resistance of Calluna-heathlands across the entire distribution range to shifting environments under global change.

Here, we evaluate the impact of different doses of $\mathrm{N}$ loading $(0,10$, 20 , and $50 \mathrm{~kg} \mathrm{Nha}^{-1} \mathrm{yr}^{-1}$ for 1 year, and $56 \mathrm{~kg} \mathrm{Nha}^{-1} \mathrm{yr}^{-1}$ for 9 years) on the functioning of Calluna-heathlands at their southern-most distribution limit by assessing the responses of two life-cycle stages of Calluna-heathland development: building/young (9-year-old plants) and mature (30-40-year-old) growth phases (Gimingham, 1972). To date, there are only a few studies testing how age affects heathland responses to $\mathrm{N}$ deposition (Caporn et al., 2014; Jones and Power, 2015; Meyer-Grünefeldt et al., 2015, 2016); this is despite contrasting plant growth rates and vegetation structures among heathland life-cycle stages (i.e., pioneer, building, mature and degenerate phases; Gimingham, 1972). Specifically, we examine how increased $\mathrm{N}$ inputs alter the annual growth and flowering of Calluna, the different plant functional groups (annual/perennial forbs and graminoids, woody species, bryophytes and lichens), and plant species composition and richness. According to prior findings from north-western and central European heathlands, we hypothesised that experimentally-increased $\mathrm{N}$ availability in rear-edge heathlands will result in: (i) enhanced Calluna-plant productivity (Bähring et al., 2017; Britton and Fisher, 2008; Southon et al., 2012) especially at the building stage due to higher plant growth rates and nutrient demand (Jones and Power, 2015); (ii) a shift in plant species composition towards more nitrophilous plant communities (Britton and Fisher, 2007; Southon et al., 2013) with higher relative abundances of graminoid species (Bobbink and Heil, 1993; Friedrich et al., 2011; Prins et al., 1991); and (iii) a decline in plant species richness (Maskell et al., 2010; Southon et al., 2013) associated to the loss of N-sensitive species (Caporn et al., 2014; Van den Berg et al., 2008) such as bryophytes and lichens (Bähring et al., 2017; Pilkington et al., 2007), particularly with cumulative (9-year) N inputs (De Schrijver et al., 2011).

\section{Material and methods}

\subsection{Study area}

The study was conducted in three Calluna heathland sites (San Isidro, Riopinos I, and Riopinos II) located in the Cantabrian Mountains (NW Spain; $43^{\circ} 02-03^{\prime} \mathrm{N}, 5^{\circ} 21-26^{\prime} \mathrm{W} ; 1560-1660$ ma.s.1.; 18-35 ha in size) [see Calvo-Fernández et al., 2018 for additional information] included as priority habitat type in Annex I of the Habitats Directive (92/43/ECC; habitat code 4060: alpine and boreal heaths). The climate is Eurosiberian (total precipitation of $1308 \mathrm{~mm}$ and mean temperature of $7.0 \pm 4.9^{\circ} \mathrm{C}$ during the study year 2014) with a brief and moderate drought period in July-August, and a snow cover duration from late-autumn until the end of May. Soils are Umbrisol characterised by high acidity $(\mathrm{pH}=3.9 \pm 0.14$; deionized water), low fertility, sandy texture and high permeability (Marcos et al., 2009). The vegetation growing season comprises from May to October and the bud burst of Calluna plants happens in June. The three sites are subjected to minimal regular free-range grazing by cattle and horses (1-2 LU ha ${ }^{-1} \mathrm{yr}^{-1}$ ) in June-September. Total $\mathrm{N}$ deposition in the study area ranges from 7.5 to $15 \mathrm{~kg} \mathrm{Nha}^{-1} \mathrm{yr}^{-1}$, according to the EMEP and CHIMERE models for Spain (García-Gómez et al., 2014), while bulk inorganic $\mathrm{N}$ deposition for the period 2011-2014 was $4.6 \mathrm{~kg} \mathrm{Nha}^{-1} \mathrm{yr}^{-1}$ (Calvo-Fernández et al., 2017). Therefore, total $\mathrm{N}$ deposition in the study area is either lower than or within the critical load range estimated to threaten the persistence of European dry $\mathrm{Cal}$ luna-heathlands (i.e., $10-20 \mathrm{~kg} \mathrm{Nha}^{-1} \mathrm{yr}^{-1}$; Bobbink et al., 2010; Hall et al., 2015).

\subsection{Experimental design}

In each study site, we selected two heathland life-cycle stages: (1) young stands rejuvenated by prescribed fire in 2005 , i.e., 9 years old at the time of the vegetation survey (building-phase; Gimingham, 1972), and (2) mature stands showing the first signs of degeneration after 30-40 years of land use abandonment (mature-phase; Gimingham, 1972). After perturbation, Calluna plants in the study area regenerate slowly and predominantly from seed germination and, to a much lesser extent, by vegetative resprouting (Calvo et al., 2002; Valbuena et al., 2000). We established a total of $902 \mathrm{~m} \times 2 \mathrm{~m}$ plots and performed a manipulative experiment consisting of five randomly-assigned $\mathrm{N}$-fertilisation treatments (i.e., 3 replicated plots per $\mathrm{N}$ treatment, age class, and site) in addition to background atmospheric $\mathrm{N}$ deposition. The yearly amount of added $\mathrm{N}$ in each treatment was: $0 \mathrm{kgNha}^{-1} \mathrm{yr}^{-1}$ (N0; control), $10 \mathrm{kgNha}^{-1} \mathrm{yr}^{-1}$ (N10), $20 \mathrm{~kg} \mathrm{Nha}^{-1} \mathrm{yr}^{-1}$ (N20), and $50 \mathrm{kgNha}^{-1} \mathrm{yr}^{-1} \quad(\mathrm{~N} 50)$ of solid granules 
of ammonium nitrate, monthly added by hand to the soil surface in June-November 2013 and 2014 (6 times in 2013, and before vegetation sampling in 2014) (short-term: 1 year); and $56 \mathrm{~kg} \mathrm{Nha}^{-1} \mathrm{yr}^{-1}$ (N56) monthly added in May-October from 2005 to 2014 (6 times per year from 2005 to 2013, and before vegetation sampling in 2014) (long-term: 9 years). The N56 treatment corresponded to ca. two times the maximum total $\mathrm{N}$ deposition levels in the study area at the beginning of the experiment (Rivero Fernández et al., 1996) and was equivalent to the highest predicted $\mathrm{N}$ input by 2050 for southern Europe (Galloway et al., 2004).

\subsection{Vegetation survey}

To evaluate the effects of the different $\mathrm{N}$-fertilisation treatments on the annual growth and flowering of Calluna plants, in each plot we measured the length and counted the number of flowers of five randomly-selected Calluna apical shoots of the current year in July 2014.

We also investigated the functional responses of the heathland vegetation to $\mathrm{N}$ fertilisation by determining the percentage cover of the different plant functional groups, the plant species composition, and the total number of plant species (species richness) in each plot in July 2014. To do this, we estimated the percentage cover of all vascular and non-vascular plant species in four $1 \mathrm{~m} \times 1 \mathrm{~m}$ quadrats in each $2 \mathrm{~m} \times 2 \mathrm{~m}$ plot, and calculated the mean percentage cover of the following plant functional groups: perennial forbs, annual forbs, perennial graminoids, annual graminoids, woody species, bryophytes, and lichens.

\subsection{Data analysis}

We fitted linear mixed models (LMMs) to test the effects of heathland age and $\mathrm{N}$ fertilisation on the annual growth and flowering of Calluna plants. The response variables in the models were mean annual Calluna shoot length and number of flowers. We modelled the response variables assuming a Gaussian error distribution, using the identity link function. The predictor variables (fixed factors) were the age of Calluna plants (young and mature), the $\mathrm{N}$ treatment (N0, N10, N20, N50, and N56), and their interaction. Minimal adequate models (MAMs) to describe the data were determined by the Akaike's information criterion (AIC) by means of the "anova ()" function. Also, we initially used the AIC to compare (a) the fit of LMMs with the identity of the sampling plots nested within each heathland site as a random factor, (b) the fit of LMMs with the identity of the heathland sites as a random factor, and (c) the fit of generalised least squares (GLS) models without the random factor, using restricted maximum likelihood estimation (REML) procedures, by means of the "anova()" and "gls()" functions. Models with the identity of the heathland sites as a random factor were the most parsimonious ones, and, therefore, the ones that are reported.

We evaluated the effects of heathland age and $\mathrm{N}$ fertilisation on total plant species richness by performing a generalised linear mixed model (GLMM) with the total number of species as the response variable, modelled following a Poisson error distribution (log link function). We also fitted analogous GLMMs with the percentage cover of the different plant functional groups: (1) perennial forbs, (2) annual forbs, (3) perennial graminoids, (4) annual graminoids, (5) woody species, (6) bryophytes, and (7) lichens, as the response variables modelled following a quasi-Poisson error distribution (log link function) to account for overdispersion. The predictor variables (fixed factors) were the age of Calluna plants, the $\mathrm{N}$ treatment, and their interaction. The interaction term was retained in the models only when significant. The identity of the sampling plots nested within each heathland site was included in the models as random factor and retained only when accounted for $>5 \%$ of the variation.

We obtained predicted values of all the response variables in the models (except for annual forbs and annual graminoids due to very low percentage cover values) for each Calluna age and $\mathrm{N}$ treatment, without taking the uncertainty of the random effects parameters into account, and computed 95\% confidence intervals based on a normal approximation. Significance levels of the difference between each $\mathrm{N}$-fertilisation treatment (N10, N20, N50, and N56) and the control treatment $(\mathrm{N} 0)$, obtained directly from the model summary outputs, are indicated in the figures.

Differences in plant species composition among $\mathrm{N}$ treatments and heathland ages were tested with non-metric multidimensional scaling (NMDS) and permutational multivariate analysis of variance (MANOVA) [999 permutations; sequential sums-of-squares (type I)] using community distance matrices and the "metaMDS()" and "adonis2()" functions. We used the Bray-Curtis dissimilarity index to calculate the site $\mathrm{x}$ species distance matrix, and evaluated the quality of the ordination with the stress value (i.e., the mismatch between distance measures and distances in the ordination space). Only species present in at least $20 \%$ of the sampling plots (i.e., 6 plots, 19 species) were included in the ordination. Predictors were the age of Calluna plants, the $\mathrm{N}$ treatment, and their interaction.

All data analyses were carried out with R software, version 3.3.1 (R Core Team, 2016) using the "stats", "MASS" (Venables and Ripley, 2002), "nlme" (Pinheiro et al., 2016), and "vegan" (Oksanen et al., 2017) packages.

\section{Results}

\subsection{CALLUNA shoot length and flowering}

The annual growth and the number of flowers of the apical shoots of both young and mature Calluna plants responded positively to the experimental increase in $\mathrm{N}$ availability (i.e., positive dose-related responses), and were significantly higher at the N20, N50, and N56 treatments compared to the control (Fig. 1, Table 1). However, we found opposite responses of annual growth and flowering to the cumulative (9-year) addition of N (N56 treatment) for each life-cycle stage of the Calluna plants (significant 'age $\mathrm{x} \mathrm{N}$ treatment' interaction terms). Relative to the rest of the $\mathrm{N}$-fertilisation treatments, the shoot length and flowering values at the N56 treatment progressively increased for mature plants, while they both decreased in case of the young ones. Moreover, young Calluna plants at the building stage generally exhibited greater annual shoot length values than mature ones.

\subsection{Plant functional groups and species composition}

The majority of plant functional groups responded statistically significantly to the experimental increase in $\mathrm{N}$ loading, especially to the cumulative (9-year) input of N (N56 treatment), and similarly for the two heathland ages (non-significant 'age $\mathrm{x} \mathrm{N}$ treatment' interaction terms) (Table 2).

The percentage cover of perennial forbs and perennial graminoids increased significantly at the N56 treatment compared to the control, for both heathland ages in case of perennial forbs, but only at mature heathlands in case of perennial graminoids (Fig. 2, Table 2). Moreover, we found significantly higher values of perennial forbs at the N10 treatment relative to the control. In general, perennial forbs exhibited low percentage cover values (young heathlands: mean \pm stan- 

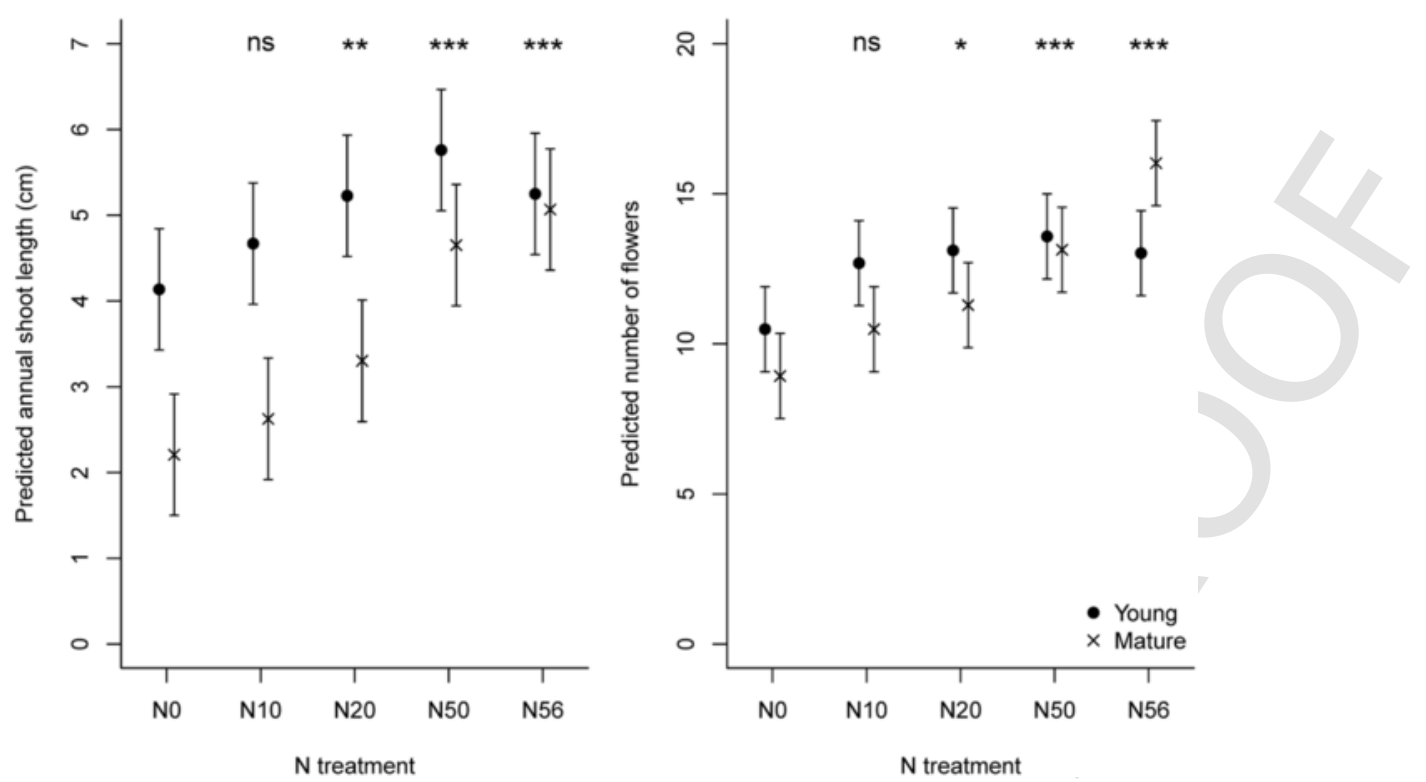

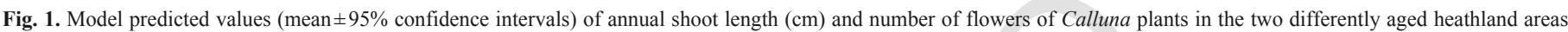

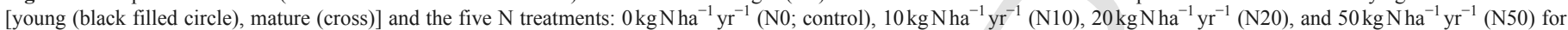

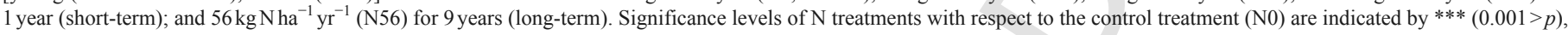
$* *(0.01>p>0.001), *(0.05>p>0.01)$, and ns $(p>0.05)$.

Table 1

Linear mixed model (LMM) results for the effects of heathland age (young, mature) and $\mathrm{N}$ treatment on Calluna annual shoot length $(\mathrm{cm})$ and number of flowers. $\mathrm{N}$ treatments: $0 \mathrm{kgNha}^{-1} \mathrm{yr}^{-1}$ (N0; control), $10 \mathrm{~kg} \mathrm{Nha}^{-1} \mathrm{yr}^{-1}$ (N10), $20 \mathrm{kgNha}^{-1} \mathrm{yr}^{-1}$ (N20), and $50 \mathrm{~kg} \mathrm{Nha}^{-1} \mathrm{yr}^{-1}$ (N50) for 1 year (short-term); and $56 \mathrm{kgNha}^{-1} \mathrm{yr}^{-1}$ (N56) for 9 years (long-term). The interaction term ('age $\mathrm{x} \mathrm{N}$ treatment') was retained in the models only when significant. Standard deviations (SD) and variance components (\%) of the random effects (identity of the heathland sites) are given. $\mathrm{Df}=$ degrees of freedom. Significant $p$-values are in bold face.

\begin{tabular}{|c|c|c|c|c|c|c|c|}
\hline & Fixed effec & & & & Random e & ects & \\
\hline $\begin{array}{l}\text { Response } \\
\text { variable }\end{array}$ & $\begin{array}{l}\text { Predictor } \\
\text { variable }\end{array}$ & Df & $\mathrm{F}$ value & $p$ value & Variable & SD & Variance \\
\hline \multirow{4}{*}{$\begin{array}{c}\text { Annual } \\
\text { shoot } \\
\text { length } \\
(\mathrm{cm})\end{array}$} & (Intercept) & 1 & 343.62 & $<0.001$ & $\begin{array}{l}\text { Heathland } \\
\text { site }\end{array}$ & 0.370 & 30.40 \\
\hline & Age & 1 & 64.81 & $<0.001$ & Residual & 0.847 & 69.60 \\
\hline & $\begin{array}{l}\mathrm{N} \\
\text { treatment }\end{array}$ & 4 & 20.43 & $<0.001$ & & & \\
\hline & $\begin{array}{l}\text { Age: } \mathrm{N} \\
\text { treatment }\end{array}$ & 4 & 3.96 & 0.006 & & & \\
\hline \multirow[t]{4}{*}{$\begin{array}{l}\text { Number of } \\
\text { flowers }\end{array}$} & (Intercept) & 1 & 1542.47 & $<0.001$ & $\begin{array}{l}\text { Heathland } \\
\text { site }\end{array}$ & 0.398 & 16.53 \\
\hline & Age & 1 & 2.03 & 0.158 & Residual & 2.010 & 83.47 \\
\hline & $\begin{array}{l}\mathrm{N} \\
\text { treatment }\end{array}$ & 4 & 14.78 & $<0.001$ & & & \\
\hline & $\begin{array}{l}\text { Age: } \mathrm{N} \\
\text { treatment }\end{array}$ & 4 & 5.00 & 0.001 & & & \\
\hline
\end{tabular}

dard deviation $=1.7 \pm 2.2$; mature heathlands: $0.8 \pm 2.0$ ); meanwhile perennial graminoids were comparatively more abundant at young $(25.4 \pm 15.5)$ than at mature heathlands $(3.9 \pm 6.5)$. The identity of the sampling plots within each heathland site (random factor in the models) explained a high amount $(>50 \%)$ of the variation in the percentage cover of perennial forbs, while a low amount $(<10 \%)$ in case of perennial graminoids (Table 2).

The percentage cover of annual forbs and annual graminoids responded significantly positively to the N56 treatment (Table 2), but reached very low average values (a single species with $<1 \%$ and $5 \%$, respectively) for both heathland ages (Tables 3 and 4). The identity of the sampling plots within each heathland site (random factor in the models) explained a very high amount $(100 \%)$ of the variation in the percentage cover of both annual forbs and annual graminoids (Table 2).

We found no significant differences in the percentage cover of woody species among the different $\mathrm{N}$-fertilisation treatments (Fig. 2, Table 2); but woody species had significantly higher cover values at the mature heathlands compared to the young ones (Fig. 2, Tables 3 and 4).

Finally, bryophytes were significantly more abundant at young heathlands, whereas lichens at mature ones (Fig. 3, Tables 3 and 4). The percentage cover values of both non-vascular plant functional groups significantly decreased at the N56 treatment relative to the control for both heathland ages (Fig. 3, Table 2).

We found no differences in plant species composition among the $\mathrm{N}$-fertilisation treatments, but we did find statistically significant differences between the two heathland ages (Fig. 4, Table 5). Young heathlands were characterised by high cover values of graminoid species such as Aira caryophyllea L., Carex nigra (L.) Reichard, Carex pilulifera L., Deschampsia flexuosa (L.) Trin., Festuca rubra L., Juncus squarrosus L., and Nardus stricta L., and bryophytes (Fig. 4, Table 3). Meanwhile mature heathlands were characterised by high cover values of woody species such as Calluna, Erica tetralix L., and Vaccinium myrtillus L., and lichens, mainly Cetraria islandica (L.) Ach. and Cladonia spp. P. Browne (Fig. 4, Table 4).

\subsection{Plant species richness}

There were no significant differences in plant species richness among the $\mathrm{N}$-fertilisation treatments for both young and mature heathlands (Fig. 5, Table 2). Then again young heathlands held a significantly higher number of plant species than mature ones. 
Table 2

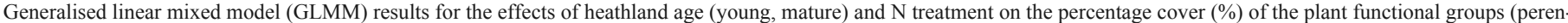

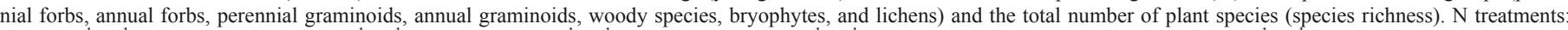

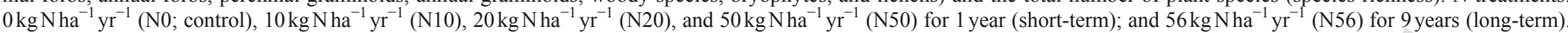

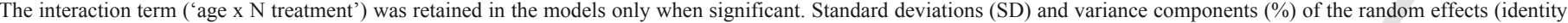
of the sampling plots nested within each heathland site) are given. $\mathrm{Df}=$ degrees of freedom. Significant $p$-values are in bold face.

\begin{tabular}{|c|c|c|c|c|c|c|c|}
\hline \multirow[b]{2}{*}{ Response variable } & \multicolumn{4}{|l|}{ Fixed effects } & \multicolumn{3}{|l|}{ Random effects } \\
\hline & Predictor variable & Df & F value & $p$ value & Variable & $\mathrm{SD}$ & Variance \\
\hline \multicolumn{8}{|l|}{ Vascular species } \\
\hline \multirow[t]{3}{*}{ Perennial forbs cover } & (Intercept) & 1 & 4.79 & 0.031 & Heathland site & 1.235 & 48.20 \\
\hline & Age & 1 & 32.54 & $<0.001$ & Sampling plot & 1.327 & 51.80 \\
\hline & $\mathrm{N}$ treatment & 4 & 2.74 & 0.034 & Residual & $1.7 \times 10^{-5}$ & $6.8 \times 10^{-4}$ \\
\hline \multirow[t]{4}{*}{ Annual forbs cover } & (Intercept) & 1 & 1447.77 & $<0.001$ & Heathland site & $1.0 \times 10^{-20}$ & 0.00 \\
\hline & Age & 1 & 11.77 & 0.001 & Sampling plot & 24.699 & 100.00 \\
\hline & $\mathrm{N}$ treatment & 4 & 6.55 & $<0.001$ & Residual & $3.2 \times 10^{-22}$ & 0.00 \\
\hline & Age: $\mathrm{N}$ treatment & 4 & 3.21 & 0.017 & & & \\
\hline \multirow[t]{4}{*}{ Perennial graminoids cover } & (Intercept) & 1 & 153.24 & $<0.001$ & Heathland site & 0.384 & 15.43 \\
\hline & Age & 1 & 60.40 & $<0.001$ & Sampling plot & 0.198 & 7.95 \\
\hline & $\mathrm{N}$ treatment & 4 & 2.70 & 0.037 & Residual & 1.909 & 76.62 \\
\hline & Age: $\mathrm{N}$ treatment & 4 & 4.21 & 0.004 & & & \\
\hline \multirow[t]{4}{*}{ Annual graminoids cover } & (Intercept) & 1 & $56,895.73$ & $<0.001$ & Heathland site & $1.1 \times 10^{-36}$ & 0.00 \\
\hline & Age & 1 & $53,522.00$ & $<0.001$ & Sampling plot & 1.387 & 100.00 \\
\hline & $\mathrm{N}$ treatment & 4 & 7912.08 & $<0.001$ & Residual & $9.1 \times 10^{-36}$ & 0.00 \\
\hline & Age: $\mathrm{N}$ treatment & 4 & 8180.93 & $<0.001$ & & & \\
\hline \multirow[t]{3}{*}{ Woody species cover } & (Intercept) & 1 & 5847.54 & $<0.001$ & Heathland site & 0.096 & 6.53 \\
\hline & Age & 1 & 62.82 & $<0.001$ & Residual & 1.368 & 93.47 \\
\hline & $\mathrm{N}$ treatment & 4 & 0.48 & 0.750 & & & \\
\hline \multicolumn{8}{|l|}{ Non-vascular species } \\
\hline \multirow[t]{3}{*}{ Bryophyte species cover } & (Intercept) & 1 & 105.80 & $<0.001$ & Heathland site & 0.344 & 13.54 \\
\hline & Age & 1 & 25.58 & $<0.001$ & Residual & 2.197 & 86.46 \\
\hline & $\mathrm{N}$ treatment & 4 & 4.68 & 0.002 & & & \\
\hline \multirow[t]{3}{*}{ Lichen species cover } & (Intercept) & 1 & 103.69 & $<0.001$ & Heathland site & 0.402 & 16.65 \\
\hline & Age & 1 & 28.75 & $<0.001$ & Sampling plot & 0.533 & 22.08 \\
\hline & $\mathrm{N}$ treatment & 4 & 8.16 & $<0.001$ & Residual & 1.479 & 61.27 \\
\hline \multirow[t]{3}{*}{ Total number of species } & (Intercept) & 1 & 553.11 & $<0.001$ & Heathland site & 0.148 & 16.53 \\
\hline & Age & 1 & 48.42 & $<0.001$ & Residual & 0.750 & 83.47 \\
\hline & $\mathrm{N}$ treatment & 4 & 1.12 & 0.352 & & & \\
\hline
\end{tabular}

\section{Discussion}

\subsection{Dose-related responses of CALLUNA plants}

The experimentally increased availability of $\mathrm{N}$ in the studied rear-edge Calluna-heathlands led to consistent dose-related responses of the annual growth and flowering of Calluna plants at both young and mature life-cycle stages. For the most part, the length as well as the number of flowers of the apical shoots of Calluna was significantly favoured by $\mathrm{N}$ fertilisation, except for young plants under cumulative (9-year) high- $\mathrm{N}\left(56 \mathrm{~kg} \mathrm{Nha}^{-1} \mathrm{yr}^{-1}\right.$ plus background deposition) loading.

Firstly, these results reflect the findings of prior studies from north-western and central European heathlands (e.g., Bähring et al., 2017; Britton and Fisher, 2008; Southon et al., 2012; Von Oheimb et al., 2010) and, thus, strongly suggest that the productivity of $\mathrm{Cal}$ luna is primarily N-limited across the entire distribution range of $\mathrm{Cal}$ luna-heathlands. However, the reported apparently positive impact of increasing $\mathrm{N}$ availability on the plant's performance requires careful consideration, because:

(1) N-driven increments in the annual growth of Calluna may entail greater plant susceptibility to a myriad of stress factors, including frost, drought (Meyer-Grünefeldt et al., 2016), and insect pests (Taboada et al., 2016). Nevertheless, no extensive Calluna plant mortality, dieback or severe damage have been detected since the beginning of the experiment in these montane heathlands (personal observations; see Calvo-Fernández et al., 2018), neither related to the cumulative loading of $\mathrm{N}$ nor caused by abiotic or biotic extreme events; oppositely to the findings reported from northern heathlands (Bjerke et al., 2017; Britton and Fisher, 2007; Carroll et al., 1999; Lee and Caporn, 1998; Log et al., 2017); and

(2) N-driven intensification of Calluna flowering may be ineffective in promoting plant reproduction and heathland regeneration, as either (i) the delay in seed germination (Valbuena et al., 2000) and the intense plant competition due to grass proliferation in young stands (Friedrich et al., 2011; Heil and Bruggink, 1987) or (ii) the absence of light (De Hullu and Gimingham, 1984) and the potential allelopathic effects on seed germination under the dense $\mathrm{Cal}$ luna canopy in mature ones (Bonanomi et al., 2005), might impede seedling recruitment (Henning et al., 2017). Consequently, further assessments of the reproductive potential of Calluna plants subjected to different $\mathrm{N}$ inputs in these rear-edge heathlands are desirable, especially if prescribed burning for heathland rejuvenation or nutrient removal is to be applied (Måren et al., 2010; Valbuena et al., 2000).

Secondly, these results evidenced the importance of undertaking long-term studies and including multiple life-history stages when assessing the response of the Calluna-heathland ecosystem to global change, partially in accordance with Jones and Power (2015) and Meyer-Grünefeldt et al. $(2015,2016)$. On the one hand, the Calluna 

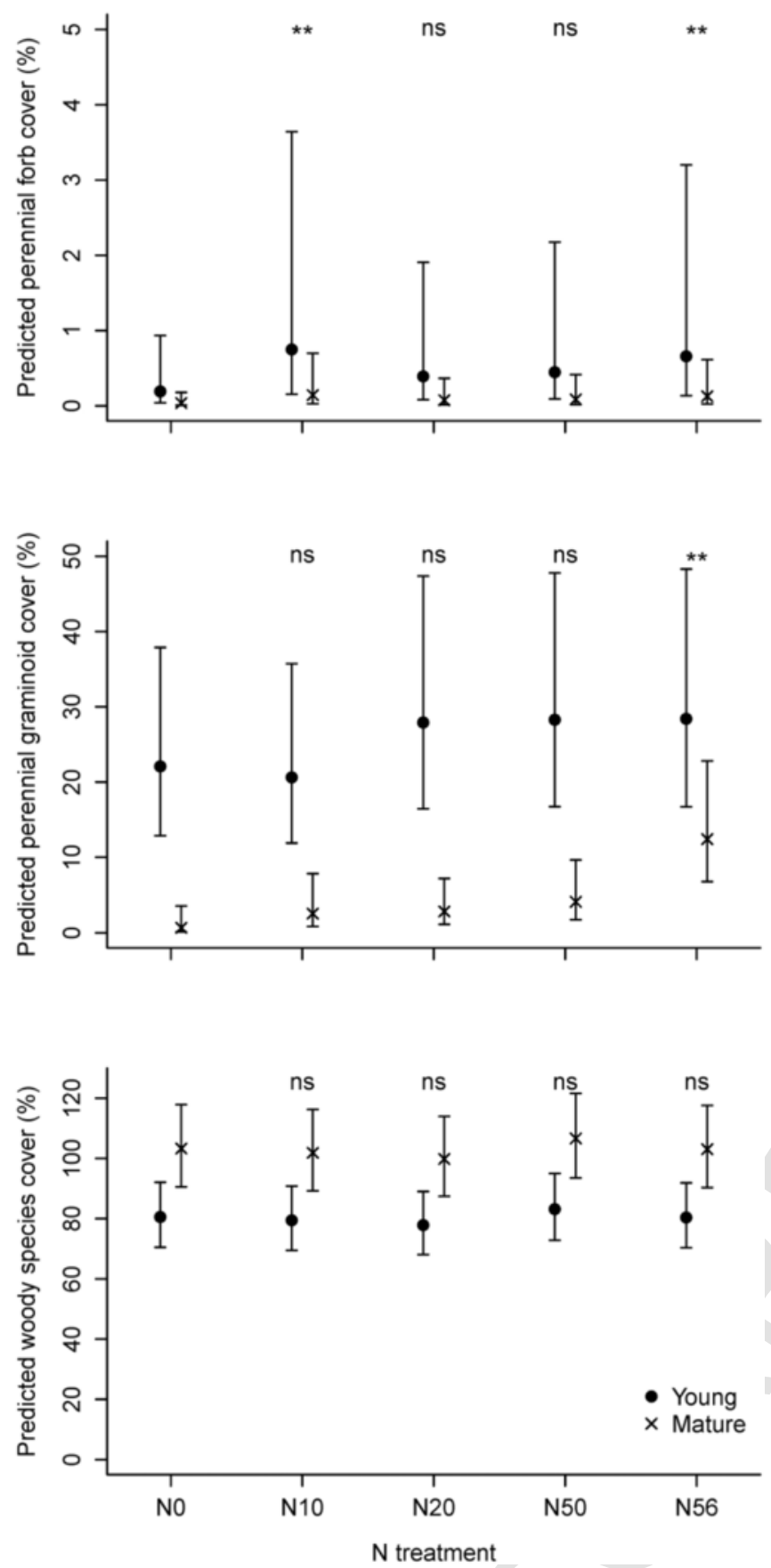

Fig. 2. Model predicted values (mean $\pm 95 \%$ confidence intervals) of the percentage cover $(\%)$ of perennial forbs, perennial graminoids, and woody species, in the two differently aged heathland areas [young (black filled circle), mature (cross)] and the five $\mathrm{N}$ treatments: $0 \mathrm{kgNha}^{-1} \mathrm{yr}^{-1}$ (N0; control), $10 \mathrm{kgNha}^{-1} \mathrm{yr}^{-1}$ (N10), $20 \mathrm{kgNha}^{-1} \mathrm{yr}^{-1}$ (N20), and $50 \mathrm{kgNha}^{-1} \mathrm{yr}^{-1}$ (N50) for 1 year (short-term); and $56 \mathrm{kgNha}^{-1} \mathrm{yr}^{-1}$ (N56) for 9 years (long-term). Significance levels of $\mathrm{N}$ treatments with respect to the control treatment $(\mathrm{N} 0)$ are indicated by $* * *(0.001>p),{ }^{* *}(0.01>p>0.001),{ }^{*}(0.05>p>0.01)$, and ns $(p>0.05)$.

plants of the surveyed montane heathlands with a short vegetation growing period during the lowest precipitation months (Calvo-Fernández et al., 2017) may rapidly uptake $\mathrm{N}$ and increase tissue $\mathrm{N}$ content right after $\mathrm{N}$ addition (Calvo-Fernández et al., 2018; "nutrient-conserving strategy" sensu Friedrich et al., 2011), but may require successive favourable growing seasons to attain noticeable
Table 3

Plant species percentage cover $(\%)$ values (mean \pm standard error) for each $\mathrm{N}$ treatment in young heathlands. $\mathrm{N}$ treatments: $0 \mathrm{~kg} \mathrm{Nha}^{-1} \mathrm{yr}^{-1}$ (N0; control), $10 \mathrm{~kg} \mathrm{Nha}^{-1} \mathrm{yr}^{-1}$ (N10), $20 \mathrm{~kg} \mathrm{Nha}^{-1} \mathrm{yr}^{-1}$ (N20), and $50 \mathrm{~kg} \mathrm{Nha}^{-1} \mathrm{yr}^{-1}$ (N50) for 1 year (short-term); and $56 \mathrm{kgNha}^{-1} \mathrm{yr}^{-1}$ (N56) for 9years (long-term). Plant functional groups: $\mathrm{AG}=$ annual graminoid, $\mathrm{PG}=$ perennial graminoid, $\mathrm{AF}=$ annual forb, $\mathrm{PF}=$ perennial forb, $\mathrm{W}=$ woody species, $\mathrm{B}=$ bryophyte, and $\mathrm{L}=$ lichen.

\begin{tabular}{|c|c|c|c|c|c|c|}
\hline & & $\mathrm{N}$ treatr & nent & & & \\
\hline & $\begin{array}{l}\text { Functional } \\
\text { group }\end{array}$ & N0 & N10 & $\mathrm{N} 20$ & N50 & N56 \\
\hline Aira caryophyllea $\mathrm{L}$. & $\mathrm{AG}$ & $\begin{array}{l}0.2 \\
(0.1)\end{array}$ & $\begin{array}{l}1.7 \\
(0.8)\end{array}$ & $\begin{array}{l}1.2 \\
(0.7)\end{array}$ & $\begin{array}{l}2.0 \\
(1.5)\end{array}$ & $\begin{array}{l}4.3 \\
(1.7)\end{array}$ \\
\hline Agrostis capillaris L. & PG & & & & $\begin{array}{l}0.2 \\
(0.2)\end{array}$ & \\
\hline Briophyta Schimp. & $\mathrm{B}$ & $\begin{array}{l}11.8 \\
(3.3)\end{array}$ & $\begin{array}{l}17.1 \\
(4.8)\end{array}$ & $\begin{array}{l}15.3 \\
(1.9)\end{array}$ & $\begin{array}{l}13.3 \\
(2.4)\end{array}$ & $\begin{array}{l}4.3 \\
(1.7)\end{array}$ \\
\hline $\begin{array}{l}\text { Calluna vulgaris (L.) } \\
\text { Hull }\end{array}$ & W & $\begin{array}{l}63.8 \\
(3.9)\end{array}$ & $\begin{array}{c}63.9 \\
(4.0)\end{array}$ & $\begin{array}{l}67.5 \\
(3.8)\end{array}$ & $\begin{array}{l}61.9 \\
(5.2)\end{array}$ & $\begin{array}{l}61.3 \\
(3.9)\end{array}$ \\
\hline Carex asturica Boissier & PG & $\begin{array}{l}0.5 \\
(0.4)\end{array}$ & $\begin{array}{l}2.1 \\
(1.5)\end{array}$ & $\begin{array}{l}1.2 \\
(0.7)\end{array}$ & $\begin{array}{l}0.4 \\
(0.4)\end{array}$ & \\
\hline $\begin{array}{l}\text { Carex nigra (L.) } \\
\text { Reichard }\end{array}$ & PG & $\begin{array}{l}1.9 \\
(1.7)\end{array}$ & $\begin{array}{l}0.5 \\
(0.4)\end{array}$ & $\begin{array}{l}1.1 \\
(1.0)\end{array}$ & $\begin{array}{l}1.9 \\
(1.2)\end{array}$ & $\begin{array}{l}2.6 \\
(1.7)\end{array}$ \\
\hline Carex pilulifera $\mathrm{L}$. & PG & $\begin{array}{l}1.8 \\
(1.0)\end{array}$ & $\begin{array}{l}1.6 \\
(0.8)\end{array}$ & $\begin{array}{l}1.8 \\
(1.0)\end{array}$ & $\begin{array}{l}2.3 \\
(1.5)\end{array}$ & $\begin{array}{l}9.4 \\
(4.4)\end{array}$ \\
\hline $\begin{array}{l}\text { Cetraria islandica }(\mathrm{L} .) \\
\text { Ach. }\end{array}$ & $\mathrm{L}$ & $\begin{array}{l}7.8 \\
(4.0)\end{array}$ & $\begin{array}{l}2.4 \\
(0.8)\end{array}$ & $\begin{array}{l}4.5 \\
(2.4)\end{array}$ & $\begin{array}{l}7.8 \\
(4.1)\end{array}$ & $\begin{array}{l}0.1 \\
(0.0)\end{array}$ \\
\hline Cladonia spp. P. Browne & $\mathrm{L}$ & $\begin{array}{l}4.8 \\
(4.0)\end{array}$ & $\begin{array}{l}0.2 \\
(0.1)\end{array}$ & $\begin{array}{l}1.1 \\
(0.5)\end{array}$ & $\begin{array}{l}4.4 \\
(1.7)\end{array}$ & $\begin{array}{l}<0.1 \\
(0.0)\end{array}$ \\
\hline $\begin{array}{l}\text { Conopodium majus } \\
\text { (Gouan) Loret. }\end{array}$ & $\mathrm{PF}$ & & $\begin{array}{l}0.1 \\
(0.1)\end{array}$ & $\begin{array}{l}0.3 \\
(0.2)\end{array}$ & & \\
\hline $\begin{array}{l}\text { Cytisus } \\
\text { oromediterraneus Rivas } \\
\text { Mart. et al. }\end{array}$ & W & $\begin{array}{l}0.4 \\
(0.4)\end{array}$ & $\begin{array}{l}1.3 \\
(1.1)\end{array}$ & $\begin{array}{l}1.3 \\
(1.3)\end{array}$ & $\begin{array}{l}0.1 \\
(0.1)\end{array}$ & \\
\hline $\begin{array}{l}\text { Daboecia cantabrica } \\
\text { (Huds.) Koch }\end{array}$ & W & $\begin{array}{l}0.1 \\
(0.1)\end{array}$ & & $\begin{array}{l}0.1 \\
(0.1)\end{array}$ & $\begin{array}{l}0.2 \\
(0.1)\end{array}$ & \\
\hline $\begin{array}{l}\text { Deschampsia flexuosa } \\
\text { (L.) Trin. }\end{array}$ & PG & $\begin{array}{l}2.0 \\
(0.6)\end{array}$ & $\begin{array}{l}2.6 \\
(0.8)\end{array}$ & $\begin{array}{l}2.8 \\
(0.5)\end{array}$ & $\begin{array}{l}2.3 \\
(0.7)\end{array}$ & $\begin{array}{l}1.5 \\
(0.6)\end{array}$ \\
\hline Erica australis L. & W & $\begin{array}{l}1.8 \\
(0.9)\end{array}$ & $\begin{array}{l}0.9 \\
(0.4)\end{array}$ & $\begin{array}{l}0.4 \\
(0.3)\end{array}$ & $\begin{array}{l}5.2 \\
(3.3)\end{array}$ & $\begin{array}{l}2.6 \\
(1.6)\end{array}$ \\
\hline Erica tetralix L. & W & $\begin{array}{l}6.4 \\
(3.7)\end{array}$ & $\begin{array}{l}1.9 \\
(1.1)\end{array}$ & $\begin{array}{l}3.1 \\
(1.4)\end{array}$ & $\begin{array}{l}11.6 \\
(3.4)\end{array}$ & $\begin{array}{l}11.3 \\
(4.8)\end{array}$ \\
\hline Festuca rubra L. & PG & $\begin{array}{l}7.4 \\
(1.4)\end{array}$ & $\begin{array}{l}7.1 \\
(2.0)\end{array}$ & $\begin{array}{l}7.3 \\
(2.9)\end{array}$ & $\begin{array}{l}2.5 \\
(1.1)\end{array}$ & $\begin{array}{l}5.0 \\
(1.9)\end{array}$ \\
\hline Galium saxatile L. & $\mathrm{PF}$ & & $\begin{array}{l}0.1 \\
(0.1)\end{array}$ & $\begin{array}{l}0.1 \\
(0.1)\end{array}$ & $\begin{array}{l}<0.1 \\
(0.0)\end{array}$ & $\begin{array}{l}0.1 \\
(0.1)\end{array}$ \\
\hline Hieracium pilosella $\mathrm{L}$. & $\mathrm{PF}$ & & $\begin{array}{l}0.2 \\
(0.2)\end{array}$ & & & \\
\hline Jasione montana L. & $\mathrm{AF}$ & & & & & $\begin{array}{l}0.1 \\
(0.1)\end{array}$ \\
\hline Juncus squarrosus L. & PG & $\begin{array}{l}2.6 \\
(1.5)\end{array}$ & $\begin{array}{l}2.8 \\
(1.2)\end{array}$ & $\begin{array}{l}4.2 \\
(2.3)\end{array}$ & $\begin{array}{l}11.4 \\
(3.1)\end{array}$ & $\begin{array}{l}6.2 \\
(2.3)\end{array}$ \\
\hline Luzula spp. DC. & PG & $\begin{array}{l}0.1 \\
(0.1)\end{array}$ & & & & \\
\hline Nardus stricta L. & PG & $\begin{array}{l}6.5 \\
(2.7)\end{array}$ & $\begin{array}{l}3.3 \\
(1.0)\end{array}$ & $\begin{array}{l}9.1 \\
(3.4)\end{array}$ & $\begin{array}{l}7.2 \\
(2.8)\end{array}$ & $\begin{array}{l}3.9 \\
(1.8)\end{array}$ \\
\hline Polygala vulgaris L. & $\mathrm{PF}$ & $\begin{array}{l}0.1 \\
(0.1)\end{array}$ & $\begin{array}{l}0.1 \\
(0.1)\end{array}$ & $\begin{array}{l}0.1 \\
(0.0)\end{array}$ & & \\
\hline $\begin{array}{l}\text { Potentilla erecta (L.) } \\
\text { Raeusch. }\end{array}$ & $\mathrm{PF}$ & $\begin{array}{l}0.1 \\
(0.1)\end{array}$ & & & $\begin{array}{l}1.3 \\
(0.7)\end{array}$ & $\begin{array}{l}0.5 \\
(0.3)\end{array}$ \\
\hline $\begin{array}{l}\text { Pterospartum } \\
\text { tridentatum } \mathrm{L} .\end{array}$ & W & $\begin{array}{l}0.1 \\
(0.1)\end{array}$ & & & & \\
\hline Rumex acetosella L. & $\mathrm{PF}$ & $\begin{array}{l}0.6 \\
(0.4)\end{array}$ & $\begin{array}{l}0.4 \\
(0.3)\end{array}$ & $\begin{array}{l}0.2 \\
(0.1)\end{array}$ & $\begin{array}{l}<0.1 \\
(0.0)\end{array}$ & $\begin{array}{l}0.6 \\
(0.3)\end{array}$ \\
\hline Scilla spp. L. & PG & & & & & $\begin{array}{c}<0.1 \\
(0.0)\end{array}$ \\
\hline Sedum album $\mathrm{L}$. & $\mathrm{PF}$ & & $\begin{array}{l}0.1 \\
(0.1)\end{array}$ & $\begin{array}{l}0.1 \\
(0.1)\end{array}$ & & \\
\hline $\begin{array}{l}\text { Thymelaea dendrobium } \\
\text { (Lam.) Endl. }\end{array}$ & $\mathrm{PF}$ & $\begin{array}{l}0.3 \\
(0.3)\end{array}$ & $\begin{array}{l}1.9 \\
(0.8)\end{array}$ & $\begin{array}{l}0.9 \\
(0.7)\end{array}$ & & \\
\hline
\end{tabular}


Table 3 (Continued)

\begin{tabular}{lllllll}
\hline \multicolumn{7}{c}{$\mathrm{N}$ treatment } \\
\hline & $\begin{array}{l}\text { Functional } \\
\text { group }\end{array}$ & $\mathrm{N} 0$ & $\mathrm{~N} 10$ & $\mathrm{~N} 20$ & $\mathrm{~N} 50$ & $\mathrm{~N} 56$ \\
\hline Vaccinium myrtillus L. & $\mathrm{W}$ & $\begin{array}{c}9.0 \\
(4.1)\end{array}$ & $\begin{array}{c}6.6 \\
(4.5)\end{array}$ & $\begin{array}{c}11.1 \\
(6.4)\end{array}$ & $\begin{array}{c}7.9 \\
(4.0)\end{array}$ & $\begin{array}{c}1.5 \\
(0.8)\end{array}$ \\
\hline
\end{tabular}

Table 4

Plant species percentage cover (\%) values (mean \pm standard error) for each $\mathrm{N}$ treatment in mature heathlands. $\mathrm{N}$ treatments: $0 \mathrm{kgNha}^{-1} \mathrm{yr}^{-1}$ (N0; control), $10 \mathrm{~kg} \mathrm{Nha}^{-1} \mathrm{yr}^{-1}$ (N10), $20 \mathrm{~kg} \mathrm{Nha}^{-1} \mathrm{yr}^{-1}$ (N20), and $50 \mathrm{~kg} \mathrm{Nha}^{-1} \mathrm{yr}^{-1}$ (N50) for 1 year (short-term); and $56 \mathrm{kgNha}^{-1} \mathrm{yr}^{-1}$ (N56) for 9years (long-term). Plant functional groups: $\mathrm{AG}=$ annual graminoid, $\mathrm{PG}=$ perennial graminoid, $\mathrm{AF}=$ annual forb, $\mathrm{PF}=$ perennial forb, $\mathrm{W}=$ woody species, $\mathrm{B}=$ bryophyte, and $\mathrm{L}=$ lichen.

\begin{tabular}{|c|c|c|c|c|c|c|}
\hline & & $\mathrm{N}$ treatr & nent & & & \\
\hline & $\begin{array}{l}\text { Functional } \\
\text { group }\end{array}$ & N0 & N10 & $\mathrm{N} 20$ & N50 & N56 \\
\hline Aira caryophyllea $\mathrm{L}$. & AG & $\begin{array}{l}<0.1 \\
(0.0)\end{array}$ & $\begin{array}{l}<0.1 \\
(0.0)\end{array}$ & & & \\
\hline Agrostis capillaris L. & PG & & & & & $\begin{array}{l}0.3 \\
(0.2)\end{array}$ \\
\hline Briophyta Schimp. & B & $\begin{array}{l}4.6 \\
(1.8)\end{array}$ & $\begin{array}{l}5.3 \\
(1.6)\end{array}$ & $\begin{array}{l}6.2 \\
(1.8)\end{array}$ & $\begin{array}{l}8.3 \\
(3.4)\end{array}$ & $\begin{array}{l}0.5 \\
(0.2)\end{array}$ \\
\hline $\begin{array}{l}\text { Calluna vulgaris (L.) } \\
\text { Hull }\end{array}$ & W & $\begin{array}{l}81.9 \\
(2.1)\end{array}$ & $\begin{array}{l}78.3 \\
(2.2)\end{array}$ & $\begin{array}{l}78.1 \\
(2.1)\end{array}$ & $\begin{array}{l}79.8 \\
(1.8)\end{array}$ & $\begin{array}{l}81.7 \\
(2.1)\end{array}$ \\
\hline $\begin{array}{l}\text { Carex nigra }(\mathrm{L} .) \\
\text { Reichard }\end{array}$ & PG & & & & $\begin{array}{l}0.4 \\
(0.4)\end{array}$ & $\begin{array}{l}<0.1 \\
(0.1)\end{array}$ \\
\hline Carex pilulifera $\mathrm{L}$. & PG & $\begin{array}{l}<0.1 \\
(0.0)\end{array}$ & & $\begin{array}{l}<0.1 \\
(0.0)\end{array}$ & & $\begin{array}{l}2.6 \\
(0.9)\end{array}$ \\
\hline $\begin{array}{l}\text { Cetraria islandica }(\mathrm{L} .) \\
\text { Ach. }\end{array}$ & $\mathrm{L}$ & $\begin{array}{l}11.0 \\
(1.6)\end{array}$ & $\begin{array}{c}10.4 \\
(1.7)\end{array}$ & $\begin{array}{l}13.3 \\
(2.2)\end{array}$ & $\begin{array}{l}13.8 \\
(3.6)\end{array}$ & $\begin{array}{l}2.0 \\
(0.9)\end{array}$ \\
\hline Cladonia spp. P. Browne & $\mathrm{L}$ & $\begin{array}{l}7.5 \\
(2.0)\end{array}$ & $\begin{array}{l}9.3 \\
(1.6)\end{array}$ & $\begin{array}{l}11.6 \\
(1.6)\end{array}$ & $\begin{array}{l}11.5 \\
(2.2)\end{array}$ & $\begin{array}{l}0.2 \\
(0.1)\end{array}$ \\
\hline $\begin{array}{l}\text { Conopodium majus } \\
\text { (Gouan) Loret. }\end{array}$ & $\mathrm{PF}$ & $\begin{array}{l}0.1 \\
(0.1)\end{array}$ & $\begin{array}{l}0.1 \\
(0.0)\end{array}$ & & & \\
\hline $\begin{array}{l}\text { Cytisus } \\
\text { oromediterraneus Rivas } \\
\text { Mart. et al. }\end{array}$ & W & $\begin{array}{l}0.1 \\
(0.1)\end{array}$ & & $\begin{array}{l}0.1 \\
(0.1)\end{array}$ & $\begin{array}{l}<0.1 \\
(0.0)\end{array}$ & \\
\hline $\begin{array}{l}\text { Daboecia cantabrica } \\
\text { (Huds.) Koch }\end{array}$ & W & & & & & \\
\hline $\begin{array}{l}\text { Deschampsia flexuosa } \\
\text { (L.) Trin. }\end{array}$ & PG & $\begin{array}{l}<0.1 \\
(0.0)\end{array}$ & $\begin{array}{l}0.1 \\
(0.1)\end{array}$ & $\begin{array}{l}0.3 \\
(0.1)\end{array}$ & $\begin{array}{l}0.3 \\
(0.2)\end{array}$ & \\
\hline Erica australis L. & W & & $\begin{array}{l}0.3 \\
(0.3)\end{array}$ & $\begin{array}{l}0.3 \\
(0.3)\end{array}$ & $\begin{array}{l}0.8 \\
(0.6)\end{array}$ & \\
\hline Erica tetralix $\mathrm{L}$. & W & $\begin{array}{l}10.7 \\
(5.2)\end{array}$ & $\begin{array}{l}16.8 \\
(8.3)\end{array}$ & $\begin{array}{l}7.0 \\
(3.6)\end{array}$ & $\begin{array}{l}13.8 \\
(7.7)\end{array}$ & 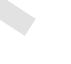 \\
\hline Festuca rubra L. & PG & $\begin{array}{l}0.4 \\
(0.2)\end{array}$ & $\begin{array}{l}1.1 \\
(0.9)\end{array}$ & $\begin{array}{l}1.4 \\
(0.3)\end{array}$ & $\begin{array}{l}1.4 \\
(0.6)\end{array}$ & \\
\hline Galium saxatile L. & $\mathrm{PF}$ & & $\begin{array}{l}0.1 \\
(0.1)\end{array}$ & $\begin{array}{l}0.1 \\
(0.1)\end{array}$ & $\begin{array}{l}0.5 \\
(0.5)\end{array}$ & \\
\hline Jasione montana L. & $\mathrm{AF}$ & & & $\begin{array}{l}0.1 \\
(0.1)\end{array}$ & $\begin{array}{l}0.2 \\
(0.1)\end{array}$ & \\
\hline Juncus squarrosus L. & PG & $\begin{array}{l}<0.1 \\
(0.0)\end{array}$ & & $\begin{array}{l}0.6 \\
(0.4)\end{array}$ & $\begin{array}{l}0.1 \\
(0.1)\end{array}$ & \\
\hline Luzula spp. DC. & PG & & & $\begin{array}{l}0.1 \\
(0.1)\end{array}$ & & \\
\hline Nardus stricta L. & PG & $\begin{array}{l}0.1 \\
(0.1)\end{array}$ & $\begin{array}{l}0.2 \\
(0.1)\end{array}$ & $\begin{array}{l}0.1 \\
(0.1)\end{array}$ & $\begin{array}{l}0.9 \\
(0.7)\end{array}$ & \\
\hline Polygala vulgaris L. & $\mathrm{PF}$ & & $\begin{array}{l}<0.1 \\
(0.0)\end{array}$ & $\begin{array}{l}<0.1 \\
(0.0)\end{array}$ & $\begin{array}{l}0.1 \\
(0.1)\end{array}$ & \\
\hline $\begin{array}{l}\text { Potentilla erecta }(\mathrm{L} .) \\
\text { Raeusch. }\end{array}$ & $\mathrm{PF}$ & & & & & \\
\hline Rumex acetosella $\mathrm{L}$. & $\mathrm{PF}$ & & & & & \\
\hline $\begin{array}{l}\text { Thymelaea dendrobium } \\
\text { (Lam.) Endl. }\end{array}$ & $\mathrm{PF}$ & & $\begin{array}{l}0.4 \\
(0.4)\end{array}$ & $\begin{array}{l}0.6 \\
(0.6)\end{array}$ & $\begin{array}{l}0.6 \\
(0.5)\end{array}$ & \\
\hline Vaccinium myrtillus L. & W & $\begin{array}{l}10.3 \\
(5.1)\end{array}$ & $\begin{array}{l}12.5 \\
(5.9)\end{array}$ & $\begin{array}{l}9.6 \\
(4.5)\end{array}$ & $\begin{array}{l}9.4 \\
(4.8)\end{array}$ & \\
\hline
\end{tabular}

$\mathrm{N}$-induced changes in biomass accumulation (Calvo et al., 2002, 2005; see also Southon et al., 2012). This idea would explain the comparatively low growth rate and small size (height: mean \pm standard deviation $=20.5 \pm 7.1 \mathrm{~cm}$; unpublished data) achieved by the young (9-year-old) Calluna plants recruited after prescribed burning at the experimental plots [percentage cover: mean \pm standard deviation $=63.7 \pm 12.2 \%$, considerably lower than the $90 \%$ established by Gimingham, 1972 at the building phase (7-13 years old)]. As well as it would explain the fact that the slow-growing mature ( $>30$-year-old) Calluna plants have not yet reached the degenerate phase [established by Gimingham, 1972 for Calluna individuals 16 to 29 or more years of age] and are, thus, still able to withstand competition with grasses (Aerts et al., 1990; Alonso and Hartley, 1998).

On the other hand, Calluna plant responses to cumulative high-N loads varied greatly with the plant's life-history stage at the studied rear-edge heathlands. Contrarily to mature Calluna plants (see Calvo-Fernández et al., 2015), young plants recovering slowly from management showed early signs of $\mathrm{N}$ saturation (sensu Aber et al., 1989) after 9 years of $\mathrm{N}$ fertilisation, with reduced annual growth and flowering despite of high $\mathrm{N}$ and phosphorous (P) foliar tissue contents (Calvo-Fernández et al., 2018). This finding would indicate that the long-term increased availability of $\mathrm{N}$ (i.e., cumulative dose of $504 \mathrm{~kg} \mathrm{Nha}^{-1}$ ) may have exceeded the plant's $\mathrm{N}$ demand and assimilation rate. This could be related to the low root development of these rear-edge Calluna plants in the early growth stages (Meyer-Grünefeldt et al., 2016) and to the increased competition for belowground resources with perennial graminoids at the experimentally-created bare soil patches (Hartley and Amos, 1999; Heil and Bruggink, 1987; Friedrich et al., 2011). It is most likely that the application of prescribed burning to rejuvenate the mature Calluna-heathland had created open spaces rapidly colonised by fast-growing graminoid species (like Carex pilulifera, Festuca rubra, Juncus squarrosus, and Nardus stricta, already present in the mature heathland) that outcompeted Calluna at the pioneer stage (see Barker et al., 2004; Hartley and Amos, 1999; Heil and Bruggink, 1987; Friedrich et al., 2011); resulting in high graminoid cover values at the building stage. This may have further implied less availability of light for young Calluna plants, a resource that might have limited Calluna plant productivity (Aerts et al., 1990), contributing to $\mathrm{N}$ saturation in this rear-edge heathland location (Aber et al., 1989).

\subsection{Vegetation and functional group responses to cumulative high-n loading}

Neither plant species composition nor species richness responded to the experimental addition of $\mathrm{N}$ in the studied rear-edge Calluna-heathlands, although major reductions in the percentage cover of non-vascular plant functional groups (i.e., bryophytes and lichens) were detected with cumulative (9-year) high-N $\left(56 \mathrm{~kg} \mathrm{Nha}^{-1} \mathrm{yr}^{-1}\right.$ plus background deposition) inputs at both young and mature life-cycle stages. Furthermore, we found low percentage cover values of annual/perennial forbs and annual graminoids at all $\mathrm{N}$-fertilisation treatments and life-cycle stages, with high within plot variability especially in young heathlands. Perennial graminoids were rather abundant in young heathlands, but did not respond to the addition of $\mathrm{N}$; in contrast with mature heathlands where they exhibited lower cover values and generally augmented with cumulative high-N loading.

It has been suggested that the lack of an N-driven effect on the heathland vegetation could be due to a prior loss of $\mathrm{N}$-sensitive species in areas with historically high-N inputs (Emmett, 2007; Soons et al., 2017). However, no remarkable decline in the number of species inhabiting the studied rear-edge heathlands has been observed 

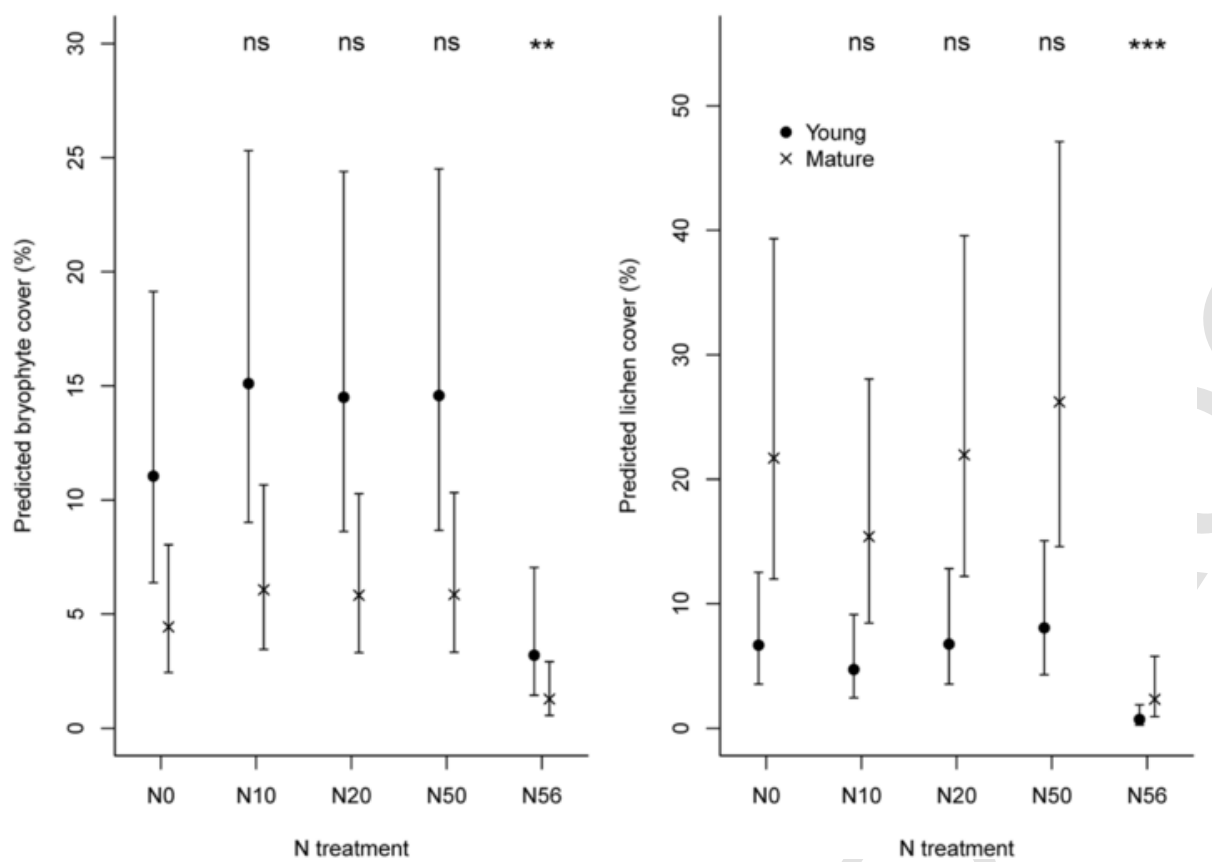

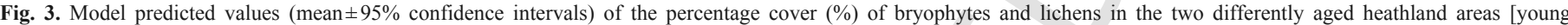

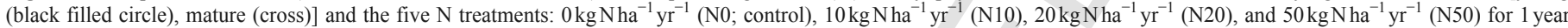

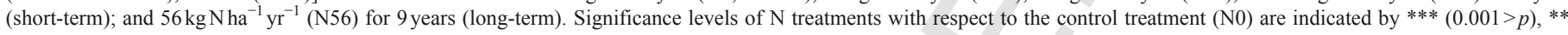
$(0.01>p>0.001), *(0.05>p>0.01)$, and ns $(p>0.05)$.

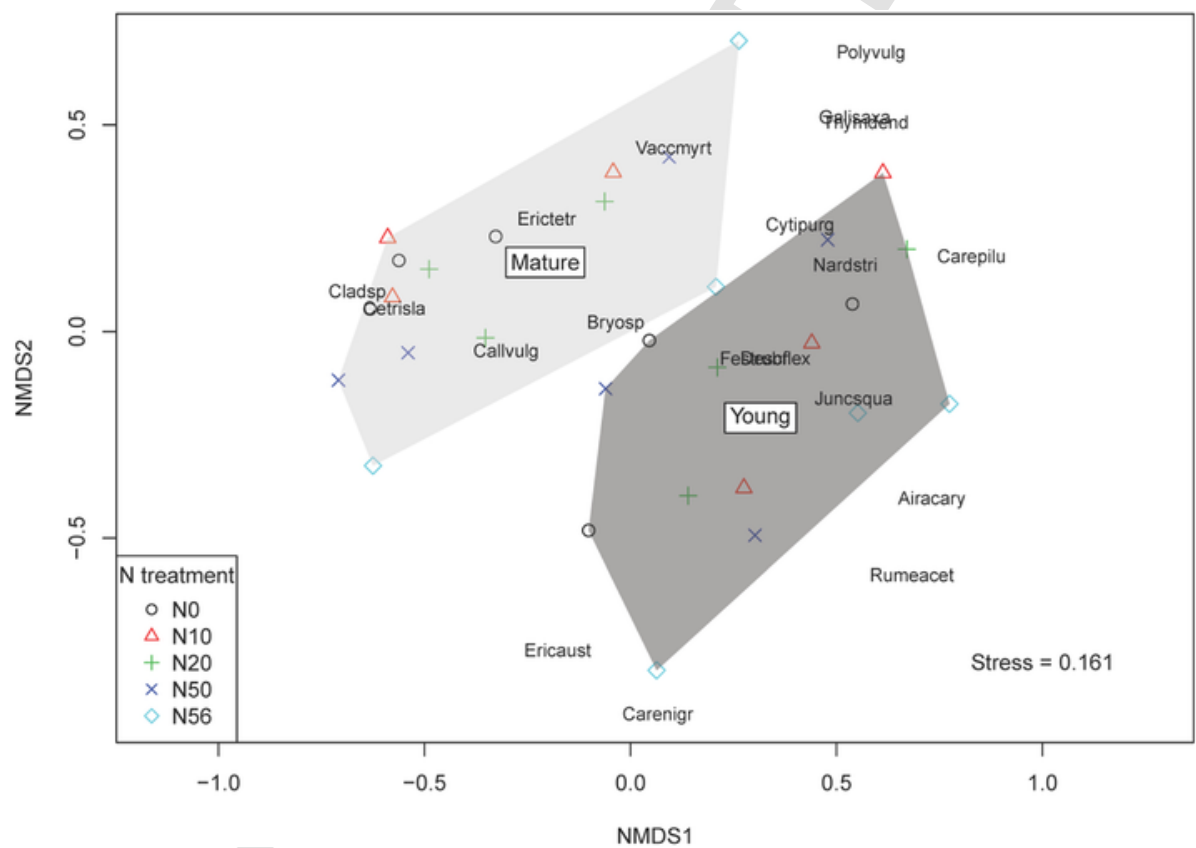

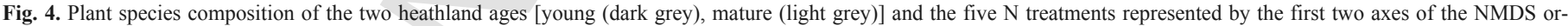

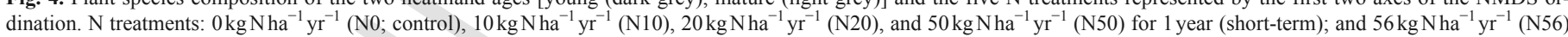

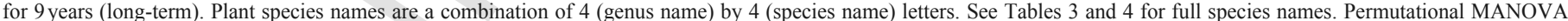
revealed significant differences in species composition between the two heathland ages (see Table 5).

since the 1980s (Calvo et al., 2002, 2005, 2007, 2012; Cuesta et al., 2008). These results may, therefore, indicate that these heathlands are moderately resistant to increased $\mathrm{N}$ availability (up to $56 \mathrm{~kg} \mathrm{Nha}^{-1} \mathrm{yr}^{-1}$ plus background deposition) in terms of vascular vegetation structure and functional composition (see Calvo et al.,
2005), and may require longer time periods ( $>10$ years) for the negative impact of high-N availability to become evident (see Calvo-Fernández et al., 2018). In contrast, north-western and central Calluna-heathlands most often experienced the loss of plant diversity (Britton and Fisher, 2007; Maskell et al., 2010; Southon et al., 2013; 
Table 5

Results of the permutational MANOVA testing for differences in plant species composition among the two heathland ages (young, mature) and the five $\mathrm{N}$ treatments: $0 \mathrm{kgNha}^{-1} \mathrm{yr}^{-1}$ (N0; control), $10 \mathrm{kgNha}^{-1} \mathrm{yr}^{-1}$ (N10), $20 \mathrm{kgNha}^{-1} \mathrm{yr}^{-1}$ (N20), and $50 \mathrm{kgNha}^{-1} \mathrm{yr}^{-1}$ (N50) for 1 year (short-term); and $56 \mathrm{kgNha}^{-1} \mathrm{yr}^{-1}$ (N56) for 9years (long-term). $\mathrm{Df}=$ degrees of freedom. Significant $\mathrm{p}$-values are in bold face.

\begin{tabular}{lllll}
\hline Factor & Df & Sum of squares & F value & $p$ value \\
\hline Age & 1 & 0.48 & 8.67 & $\mathbf{0 . 0 0 1}$ \\
N treatment & 4 & 0.17 & 0.78 & 0.698 \\
Age: N treatment & 4 & 0.06 & 0.28 & 0.995 \\
Residual & 20 & 1.11 & & \\
\hline
\end{tabular}

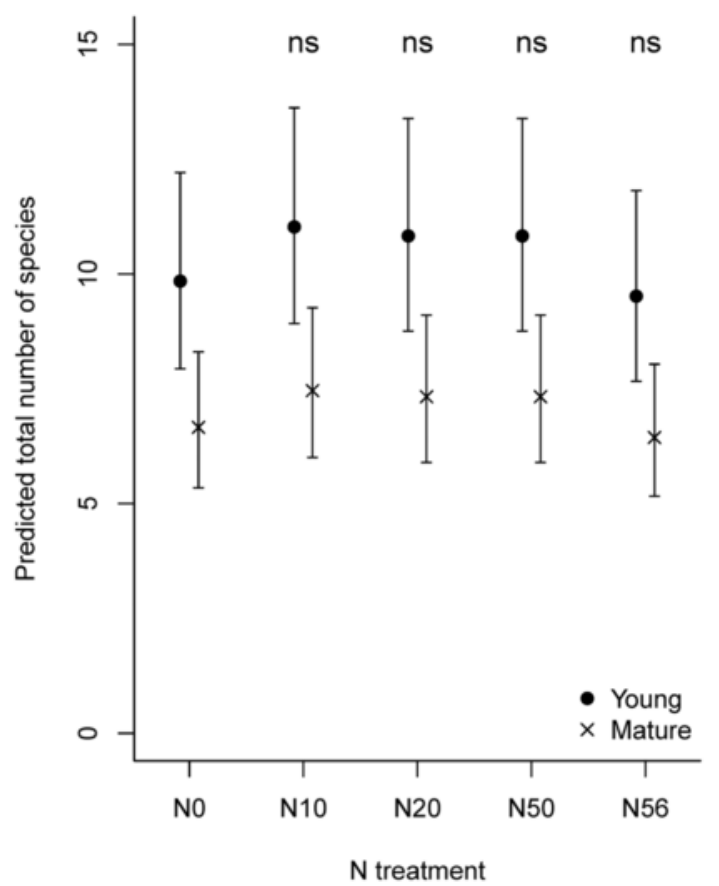

Fig. 5. Model predicted values (mean $\pm 95 \%$ confidence intervals) of plant species richness in the two differently aged heathland areas [young (black filled circle), mature (cross)] and the five $\mathrm{N}$ treatments: $0 \mathrm{~kg} \mathrm{Nha}^{-1} \mathrm{yr}^{-1}$ (N0; control), $10 \mathrm{~kg} \mathrm{Nha}^{-1} \mathrm{yr}^{-1}$ (N10), $20 \mathrm{~kg} \mathrm{Nha}^{-1} \mathrm{yr}^{-1}$ (N20), and $50 \mathrm{~kg} \mathrm{Nha}^{-1} \mathrm{yr}^{-1}$ (N50) for 1 year (short-term); and $56 \mathrm{kgNha}^{-1} \mathrm{yr}^{-1}$ (N56) for 9years (long-term). Significance levels of $\mathrm{N}$ treatments with respect to the control treatment $(\mathrm{N} 0)$ are indicated by $* * *(0.001>p)$, ** $(0.01>p>0.001), *(0.05>p>0.01)$, and ns $(p>0.05)$.

see meta-analysis by De Schrijver et al., 2011) and pronounced changes in plant species dominance (Aerts et al., 1990; Bobbink and Heil, 1993; Heil and Diemont, 1983; Prins et al., 1991) under elevated $\mathrm{N}$ loading, generally linked to an increased Calluna susceptibility to environmental stresses (e.g., herbivory, frost, and winter drought).

The observed negative responses of bryophytes and lichens to cumulative (9-year) high-N (56 kg Nha ${ }^{-1} \mathrm{yr}^{-1}$ plus background $\mathrm{N}$ deposition) loading in our rear-edge Calluna-heathlands are consistent with many previous studies from north-western and central European heathlands (e.g., Bähring et al., 2017; Caporn et al., 2014; Southon et al., 2013). In these heathlands non-vascular species reacted faster and more sensitive than vascular plants to increased $\mathrm{N}$ deposition (e.g., 2 years, $5 \mathrm{~kg} \mathrm{Nha}^{-1} \mathrm{yr}^{-1}$ : Bähring et al., 2017; 4 years, $10 \mathrm{~kg} \mathrm{Nha}^{-1} \mathrm{yr}^{-1}$ : Britton and Fisher, 2007; 4 years, $10 \mathrm{kgNha}^{-1} \mathrm{yr}^{-1}$ : Pilkington et al., 2007). Contrarily to these studies, however, we only found significant reductions in the percentage cover of both cryp- togam functional groups after many years of $\mathrm{N}$ fertilisation and under high-N inputs (i.e., cumulative dose of $504 \mathrm{~kg} \mathrm{Nha}^{-1}$; see De Schrijver et al., 2011). This likely indicates that the rate of change due to enhanced $\mathrm{N}$ availability in the percentage cover of non-vascular vegetation of rear-edge Calluna-heathlands is slow and/or requires greater doses of $\mathrm{N}$ deposition in comparison to north-western and central heathlands. This again suggests that Calluna-heathlands at their southern-most distribution limit are relatively more resistant to or cope better with increased $\mathrm{N}$ availability also regarding non-vascular vegetation structure.

Alternatively, the absence of broad N-driven responses of vascular and non-vascular plants to enhanced $\mathrm{N}$-availability could be the result of either an effect of the experimental design (i.e., $2 \mathrm{~m} \times 2 \mathrm{~m}$ plot-based field experiment with considerable variation among plots), an effect of the short-term (1-year) duration of the $\mathrm{N}$-fertilisation treatments (N10, N20, and N50), or both. However, this might not be the case, as prior $\mathrm{N}$-addition plot-based experiments at these rear-edge montane heathlands detected significant changes in vascular plant species richness and percentage cover over the short-term (i.e., $1 \mathrm{~m} \times 1 \mathrm{~m}$ experimental plots, 3 months to 2 years; Calvo et al., 2005, 2007; Cuesta et al., 2008). Similarly, previous N-fertilisation plot-based experiments in Calluna-heathlands (e.g., Bähring et al., 2017; Britton and Fisher, 2007; Pilkington et al., 2007) and grassland ecosystems (e.g., Clark and Tilman, 2008; Reich, 2009; Van den Berg et al., 2011) reported significant effects of increased $\mathrm{N}$ loading on plant species richness, composition and/or functional groups over different time scales.

\subsection{Implications for heathland management at the rear-edge of the ECOSYSTEM'S distribution}

Similarly to most Calluna-heathlands across Europe (Webb, 1998), the studied rear-edge heathlands have historically been maintained by frequent small-scale perturbations, mainly controlled burning and cutting to create pastureland for breeding livestock. In the last few decades, the decline or cessation of these practices has led to the senescence of Calluna plants which might eventually reach a degenerate stage and lose their ability to outcompete grasses and encroached trees/shrubs (Betula pubescens Ehrh., Pinus sylvestris L., and Cytisus oromediterraneus Rivas Mart. et al.); as happened in other north-western, central and southern heathlands (e.g., Bartolomé et al., 2005; Rose et al., 2000). Prior experimental studies comparing different management alternatives in the surveyed rear-edge heathlands supported repeated burning on a 15-20-year rotation to prevent the degenerate phase from occurring (Calvo et al., 2002, 2012). However, our findings further suggest that rejuvenation by prescribed burning alone may be ineffective to preserve these heathlands under elevated airborne $\mathrm{N}$ deposition, as fire promoted invasion by graminoid species in our experimental plots at the pioneer phase (see Barker et al., 2004; Friedrich et al., 2011; Hartley and Amos, 1999; Heil and Bruggink, 1987). It would be therefore necessary to complement regular ( $>30$-year; see Velle et al., 2012) burning with livestock grazing at moderately-low stocking rates (Pakeman et al., 2003) before the building stage is attained (i.e., <5 years after fire; Gimingham, 1972), allowing Calluna to outcompete graminoids for light (Alonso and Hartley, 1998). Nonetheless, the most suitable fire and grazing intensities, frequencies and extents to minimise the impact of $\mathrm{N}$ accumulation should be determined by future research, especially regarding the most sensitive species (i.e., bryophytes and lichens). 


\section{Conclusions}

To our knowledge, this is the first study revealing that Calluna-heathlands at the low-latitude distribution limit are fairly resistant to cumulative (9-year) high-N $\left(56 \mathrm{~kg} \mathrm{Nha}^{-1} \mathrm{yr}^{-1}\right.$ plus background deposition) inputs, at both the building/young and mature growth stages, regarding plant functional groups relative abundances, species composition and richness. The general lack of $\mathrm{N}$-driven responses of the studied rear-edge heathlands to the long-term experimental addition of $\mathrm{N}$ diverges within the ecosystem's distribution range, as north-western and central European heathlands are broadly very sensitive to enhanced $\mathrm{N}$ availability. Furthermore, we reported (1) dose-related responses of Calluna annual growth and flowering to the addition of $\mathrm{N}$ (1 year, 10, 20, and $50 \mathrm{~kg} \mathrm{Nha}^{-1} \mathrm{yr}^{-1}$ ); (2) early signs of $\mathrm{N}$ saturation of young Calluna plants; and (3) a marked reduction in the percentage cover of bryophytes and lichens only with very high doses of $\mathrm{N}$ (i.e., cumulative dose of $504 \mathrm{~kg} \mathrm{Nha}^{-1}$ ). These results potentially indicate that rear-edge montane heathlands may be of vital importance to unravel the mechanisms of heathland ecosystem resilience (Meyer-Grünefeldt et al., 2016) under the uncertainty of global changes in the upcoming decades ( $\mathrm{N}$ fertilisation effects vs. warming and drought effects; Peñuelas et al., 2017).

\section{Acknowledgements}

We thank E. de Luis Calabuig, W. Härdtle, R. Tárrega, and L. Valbuena for collaboration. This study was financed by the Spanish Ministry of Science and Technology (research project CGL2006-10998-CO2-01/BOS) and the Regional Government of Castilla and León (research projects LE021A08 and LE039A09). J.C-F. was funded by the Spanish Ministry of Education (predoctoral fellowship FPU12/01494). Finally, we thank the reviewers for their constructive comments.

\section{References}

Aber, J.D., Nadelhoffer, K.J., Steudler, P., Melillo, J.M., 1989. Nitrogen saturation in northern forest ecosystems. Bioscience 39, 378-386.

Aerts, R., Berendse, F., de Caluwe, H., Schmitz, M., 1990. Competition in heathland along an experimental gradient of nutrient availability. Oikos 57, 310-318. https:// doi.org/10.2307/3565959.

Alonso, I., Hartley, S.E., 1998. Effects of nutrient supply, light availability and herbivory on the growth of heather and three competing grass species. Plant Ecol. 137, 203-212. https://doi.org/10.1023/A:1009770313618.

Bähring, A., Fichtner, A., Ibe, K., Schütze, G., Temperton, V.M., von Oheimb, G. Härdtle, W., 2017. Ecosystem functions as indicators for heathland responses to nitrogen fertilisation. Ecol. Indic. 72, 185-193. https://doi.org/10.1016/j.ecolind. 2016.08.013.

Barker, C.G., Power, S.A., Bell, J.N.B., Orme, C.D.L., 2004. Effects of habitat management on heathland response to atmospheric nitrogen deposition. Biol. Conserv. 120, 41-52. https://doi.org/10.1016/j.biocon.2004.01.024.

Bartolomé, J., Plaixats, J., Fanlo, R., Boada, M., 2005. Conservation of isolated Atlantic heathlands in the Mediterranean region: effects of land-use changes in the Montseny biosphere reserve (Spain). Biol. Conserv. 122, 81-88. https://doi.org/10. 1016/j.biocon.2004.05.024.

Bjerke, J.W., Treharne, R., Vikhamar-Schuler, D., Karlsen, S.R., Ravolainen, V., Bokhorst, S., Phoenix, G.K., Bochenek, Z., Tømmervik, H., 2017. Understanding the drivers of extensive plant damage in boreal and Arctic ecosystems: insights from field surveys in the aftermath of damage. Sci. Total Environ. 599-600, 1965-1976. https://doi.org/10.1016/j.scitotenv.2017.05.050.

Bobbink, R., Heil, G.W., 1993. Atmospheric deposition of sulphur and nitrogen in heathland ecosystems. In: Aerts, R., Heil, G.W. (Eds.), Heathlands: Patterns and Processes in a Changing Environment. Kluwer Academic Publishers, The Netherlands, pp. 25-50.

Bobbink, R., Hicks, K., Galloway, J., Spranger, T., Alkemade, R., Ashmore, M., Bustamante, M., Cinderby, S., Davidson, E., Dentener, F., Emmett, B., Erisman, J.-W. Fenn, M., Gilliam, F., Nordin, A., Pardo, L., de Vries, W., 2010. Global assessment of nitrogen deposition effects on terrestrial plant diversity: a synthesis. Ecol. Appl. 20, 30-59. https://doi.org/10.1890/08-1140.1.

Bonanomi, G., Legg, C., Mazzoleni, S., 2005. Autoinhibition of germination and seedling establishment by leachate of Calluna vulgaris leaves and litter. Community Ecol. 6, 203-208. https://doi.org/10.1556/ComEc.6.2005.2.8.

Britton, A.J., Fisher, J.M., 2007. Interactive effects of nitrogen deposition, fire and grazing on diversity and composition of low-alpine prostrate Calluna vulgaris heathland. J. Appl. Ecol. 44, 125-135. https://doi.org/10.1111/j.1365-2664.2006. 01251.x.

Britton, A.J., Fisher, J.M., 2008. Growth responses of low-alpine dwarf-shrub heath species to nitrogen deposition and management. Environ. Pollut. 153, 564-573. https://doi.org/10.1016/j.envpol.2007.09.022.

Britton, A.J., Hester, A.J., Hewison, R.L., Potts, J.M., Ross, L.C., 2017. Climate, pollution and grazing drive long-term change in moorland habitats. Appl. Veg. Sci. 20, 194-203. https://doi.org/10.1111/avsc. 12260.

Calvo, L., Tárrega, R., Luis, E., 2002. Regeneration patterns in a Calluna vulgaris heathland in the Cantabrian mountains (NW Spain): effects of burning, cutting and ploughing. Acta Oecol. 23, 81-90. https://doi.org/10.1016/ S1146-609X(02)01137-2

Calvo, L., Alonso, I., Fernández, A.J., De Luis, E., 2005. Short-term study of effects of fertilisation and cutting treatments on the vegetation dynamics of mountain heathlands in Spain. Plant Ecol. 179, 181-191. https://doi.org/10.1007/ s11258-004-7511-3.

Calvo, L., Alonso, I., Marcos, E., De Luis, E., 2007. Effects of cutting and nitrogen deposition on biodiversity in Cantabrian heathlands. Appl. Veg. Sci. 10, 43-52. https://doi.org/10.1658/1402-2001(2007)10[43:EOCAND]2.0.CO;2.

Calvo, L., Tárrega, R., Luis, E., 2012. Changes of species richness in heathland communities over 15 years following disturbances. Int. J. For. Res. 2012, 1-12. https:// doi.org/10.1155/2012/547120.

Calvo-Fernández, J., Marcos, E., Calvo, L., Härdtle, W., 2015. Allocation patterns of airborne nitrogen in mountainous heathlands- $\mathrm{A}^{15} \mathrm{~N}$ tracer study in the Cantabrian Mountains (NW Spain). Ecol. Eng. 84, 128-135. https://doi.org/10.1016/j.ecoleng. 2015.07.027

Calvo-Fernández, J., Marcos, E., Calvo, L., 2017. Bulk deposition of atmospheric inorganic nitrogen in mountainous heathland ecosystems in North-Western Spain. Atmos. Res. 183, 237-244. https://doi.org/10.1016/j.atmosres.2016.09.006.

Calvo-Fernández, J., Taboada, A., Fichtner, A., Härdtle, W., Calvo, L., Marcos, E., 2018. Time- and age-related effects of experimentally simulated nitrogen deposition on the functioning of montane heathland ecosystems. Sci. Total Environ. 613-614, 149-159. https://doi.org/10.1016/j.scitotenv.2017.08.307.

Caporn, S.J.M., Carroll, J.A., Dise, N.B., Payne, R.J., 2014. Impacts and indicators of nitrogen deposition in moorlands: results from a national pollution gradient study. Ecol. Indic. 45, 227-234. https://doi.org/10.1016/j.ecolind.2014.04.019.

Carroll, J.A., Caporn, S.J.M., Cawley, L., Read, D.J., Lee, J.A., 1999. The effect of increased deposition of atmospheric nitrogen on Calluna vulgaris in upland Britain. New Phytol. 141, 423-431.

Clark, C.M., Tilman, D., 2008. Loss of plant species after chronic low-level nitrogen deposition to prairie grasslands. Nature 451, 712-715. https://doi.org/10.1038/ nature 06503 .

Cuesta, D., Taboada, A., Calvo, L., Salgado, J.M., 2008. Short- and medium-term effects of experimental nitrogen fertilization on arthropods associated with Calluna vulgaris heathlands in north-West Spain. Environ. Pollut. 152, 394-402. https:// doi.org/10.1016/j.envpol.2007.06.073.

De Hullu, E., Gimingham, C.H., 1984. Germination and establishment of seedlings in different phases of the Calluna life cycle in a Scottish heathland. Vegetatio 58, $115-121$.

De Schrijver, A., De Frenne, P., Ampoorter, E., Van Nevel, L., Demey, A., Wuyts, K., Verheyen, K., 2011. Cumulative nitrogen input drives species loss in terrestrial ecosystems. Glob. Ecol. Biogeogr. 20, 803-816. https://doi.org/10.1111/j. 1466-8238.2011.00652.x.

Emmett, B.A., 2007. Nitrogen saturation of terrestrial ecosystems: some recent findings and their implications for our conceptual framework. Water Air Soil Pollut. 7, 99-109. https://doi.org/10.1007/s11267-006-9103-9.

Fagúndez, J., 2013. Heathlands confronting global change: drivers of biodiversity loss from past to future scenarios. Ann. Bot. 111, 151-172. https://doi.org/10.1093/aob/ $\operatorname{mes} 257$.

Field, C.D., Dise, N.B., Payne, R.J., Britton, A.J., Emmett, B.A., Helliwell, R.C., Hughes, S., Jones, L., Lees, S., Leake, J.R., Leith, I.D., Phoenix, G.K., Power, S.A., Sheppard, L.J., Southon, G.E., Stevens, C.J., Caporn, S.J.M., 2014. The role of nitrogen deposition in widespread plant community change across semi-natural habitats. Ecosystems 17, 864-877. https://doi.org/10.1007/s10021-014-9765-5.

Field, C.D., Evans, C.D., Dise, N.B., Hall, J.R., Caporn, S.J.M., 2017. Long-term nitrogen deposition increases heathland carbon sequestration. Sci. Total Environ. 592, 426-435. https://doi.org/10.1016/j.scitotenv.2017.03.059.

Friedrich, U., von Oheimb, G., Dziedek, C., Kriebitzsch, W.-U., Selbmann, K., Härdtle, W., 2011. Mechanisms of purple moor-grass (Molinia caerulea) encroachment 
in dry heathland ecosystems with chronic nitrogen inputs. Environ. Pollut. 159, 3553-3559. https://doi.org/10.1016/j.envpol.2011.08.010.

Galloway, J.N., Dentener, F.J., Capone, D.G., Boyer, E.W., Howarth, R.W., Seitzinger, S.P., Asner, G.P., Cleveland, C.C., Green, P.A., Holland, E.A., Karl, D.M., Michaels, A.F., Porter, J.H., Townsend, A.R., Vörösmarty, C.J., 2004. Nitrogen cycles: past, present, and future. Biogeochemistry 70, 153-226.

Galloway, J.N., Townsend, A.R., Erisman, J.W., Bekunda, M., Cai, Z., Freney, J.R., Martinelli, L.A., Seitzinger, S.P., Sutton, M.A., 2008. Transformation of the nitrogen cycle: recent trends, questions, and potential solutions. Science 320, 889-892. https://doi.org/10.1126/science.1136674.

García-Gómez, H., Garrido, J.L., Vivanco, M.G., Lassaletta, L., Rábago, I., Àvila, A., Tsyro, S., Sánchez, G., González Ortiz, A., González-Fernández, I., Alonso, R., 2014. Nitrogen deposition in Spain: modeled patterns and threatened habitats within the Natura 2000 network. Sci. Total Environ. 485-486, 450-460. https://doi. org/10.1016/j.scitotenv.2014.03.112.

Gimingham, C.H., 1972. Ecology of Heathlands. Chapman and Hall, London, UK

Hall, J., Curtis, C., Dore, T., Smith, R., 2015. Methods for the Calculation of Critical Loads and their Exceedances in the UK. NERC/Centre for Ecology \& Hydrology, Bangor, UK.

Hampe, A., Petit, R.J., 2005. Conserving biodiversity under climate change: the rear edge matters. Ecol. Lett. 8, 461-467. https://doi.org/10.1111/j.1461-0248.2005. 00739.x.

Härdtle, W., von Oheimb, G., Gerke, A.-K., Niemeyer, M., Niemeyer, T., Assmann, T., Drees, C., Matern, A., Meyer, H., 2009. Shifts in N and P budgets of heathland ecosystems: effects of management and atmospheric inputs. Ecosystems 12, 298-310. https://doi.org/10.1007/s10021-008-9223-3.

Hartley, S.E., Amos, L., 1999. Competitive interactions between Nardus stricta L. and Calluna vulgaris (L.) Hull: the effect of fertilizer and defoliation on above- and below-ground performance. J. Ecol. 87, 330-340. https://doi.org/10.1046/j. 1365-2745.1999.00353.x

Heil, G.W., Bruggink, M., 1987. Competition for nutrients between Calluna vulgaris (L.) Hull and Molinia caerulea (L.) Moench. Oecologia 73, 105-107.

Heil, G.W., Diemont, W.H., 1983. Raised nutrient levels change heathland into grassland. Vegetatio 53, 113-120.

Henning, K., von Oheimb, G., Härdtle, W., Fichtner, A., Tischew, S., 2017. The reproductive potential and importance of key management aspects for successful Calluna vulgaris rejuvenation on abandoned continental heaths. Ecol. Evol. 7, 2091-2100. https://doi.org/10.1002/ece3.2816.

Humbert, J.-Y., Dwyer, J.M., Andrey, A., Arlettaz, R., 2016. Impacts of nitrogen addition on plant biodiversity in mountain grasslands depend on dose, application duration and climate: a systematic review. Glob. Chang. Biol. 22, 110-120. https://doi. org/10.1111/gcb.12986.

Jones, A.G., Power, S.A., 2015. Functional relationships with $\mathrm{N}$ deposition differ according to stand maturity in Calluna-dominated heathland. Ambio 44, 131-141. https://doi.org/10.1007/s13280-014-0529-4

Jones, L., Stevens, C., Rowe, E.C., Payne, R., Caporn, S.J.M., Evans, C.D., Field, C., Dale, S., 2017. Can on-site management mitigate nitrogen deposition impacts in non-wooded habitats?. Biol. Conserv. 212, 464-475. https://doi.org/10.1016/j. biocon.2016.06.012

Lee, J.A., Caporn, S.J.M., 1998. Ecological effects of atmospheric reactive nitrogen deposition on semi-natural terrestrial ecosystems. New Phytol. 139, 127-134.

Log, T., Thuestad, G., Velle, L.G., Khattri, S.K., Kleppe, G., 2017. Unmanaged heathland - a fire risk in subzero temperatures?. Fire Saf. J. 90, 62-71. https://doi.org/ 10.1016/j.firesaf.2017.04.017

Loidi, J., Biurrun, I., Campos, J.A., García-Mijangos, I., Herrera, M., 2010. A biogeographical analysis of the European Atlantic lowland heathlands. J. Veg. Sci. 21, 832-842. https://doi.org/10.1111/j.1654-1103.2010.01204.x.

Marcos, E., Calvo, L., Luis-Calabuig, E., 2003. Effects of fertilization and cutting on the chemical composition of vegetation and soils of mountain heathlands in Spain. J. Veg. Sci. 14, 417-424. https://doi.org/10.1658/1100-9233(2003)014[0417: EOFACO $2.0 . \mathrm{CO} ; 2$

Marcos, E., Villalón, C., Calvo, L., Luis-Calabuig, E., 2009. Short-term effects of experimental burning on soil nutrients in the Cantabrian Mountains. Ecol. Eng. 35, 820-828. https://doi.org/10.1016/j.ecoleng.2008.12.011.

Måren, I.E., Janovský, Z., Spindelböck, J.P., Daws, M.I., Kaland, P.E., Vandvik, V., 2010. Prescribed burning of northern heathlands: Calluna vulgaris germination cues and seed-bank dynamics. Plant Ecol. 207, 245-256. https://doi.org/10.1007/ s11258-009-9669-1.

Maskell, L.C., Smart, S.M., Bullock, J.M., Thompson, K., Stevens, C.J., 2010. Nitrogen deposition causes widespread loss of species richness in British habitats. Glob. Chang. Biol. 16, 671-679. https://doi.org/10.1111/j.1365-2486.2009.02022.x.

Meyer-Grünefeldt, M., Calvo, L., Marcos, E., von Oheimb, G., Härdtle, W., 2015. Impacts of drought and nitrogen addition on Calluna heathlands differ with plant life-history stage. J. Ecol. 103, 1141-1152. https://doi.org/10.1111/1365-2745 12446

Meyer-Grünefeldt, M., Belz, K., Calvo, L., Marcos, E., von Oheimb, G., Härdtle, W., 2016. Marginal Calluna populations are more resistant to climate change, but not under high-nitrogen loads. Plant Ecol. 217, 111-122. https://doi.org/10.1007/ s11258-015-0563-8.

Ochoa-Hueso, R., Allen, E.B., Branquinho, C., Cruz, C., Dias, T., Fenn, M.E., Manrique, E., Pérez-Corona, M.E., Sheppard, L.J., Stock, W.D., 2011. Nitrogen deposition effects on Mediterranean-type ecosystems: an ecological assessment. Environ. Pollut. 159, 2265-2279. https://doi.org/10.1016/j.envpol.2010.12.019.

Oksanen, J., Blanchet, F.G., Friendly, M., Kindt, R., Legendre, P., McGlinn, D., Minchin, P.R., O'Hara, R.B., Simpson, G.L., Solymos, P., Stevens, M.H.H., Szoecs, E., Wagner, H., 2017. vegan: Community Ecology Package. R Package Version. 2, 4, Available at https://CRAN.R-project.org/package=vegan.

Pakeman, R.J., Hulme, P.D., Torvell, L., Fisher, J.M., 2003. Rehabilitation of degraded dry heather [Calluna vulgaris (L.) hull] moorland by controlled sheep grazing. Biol. Conserv. 114, 389-400. https://doi.org/10.1016/S0006-3207(03)00067-3.

Payne, R.J., Dise, N.B., Field, C.D., Dore, A.J., Caporn, S.J.M., Stevens, C.J., 2017. Nitrogen deposition and plant biodiversity: past, present, and future. Front. Ecol. Environ. 15, 431-436. https://doi.org/10.1002/fee.1528.

Pearson, G.A., Lago-Leston, A., Mota, C., 2009. Frayed at the edges: selective pressure and adaptive response to abiotic stressors are mismatched in low diversity edge populations. J. Ecol. 97, 450-462. https://doi.org/10.1111/j.1365-2745.2009. 01481.x.

Peñuelas, J., Ciais, P., Canadell, J.G., Janssens, I.A., Fernández-Martínez, M., Carnicer, J., Obersteiner, M., Piao, S., Vautard, R., Sardans, J., 2017. Shifting from a fertilization-dominated to a warming-dominated period. Nat. Ecol. Evol. 1, 1438-1445. https://doi.org/10.1038/s41559-017-0274-8.

Phoenix, G.K., Emmett, B.A., Britton, A.J., Caporn, S.J.M., Dise, N.B., Helliwell, R., Jones, L., Leake, J.R., Leith, I., Sheppard, L.J., Sowerby, A., Pilkington, M.G., Rowe, E., Ashmore, M.R., Power, S.A., 2012. Impacts of atmospheric nitrogen deposition: responses of multiple plant and soil parameters across contrasting ecosystems in long-term field experiments. Glob. Chang. Biol. 18, 1197-1215. https:// doi.org/10.1111/j.1365-2486.2011.02590.x.

Pilkington, M.G., Caporn, S.J.M., Carroll, J.A., Cresswell, N., Lee, J.A., Emmett, B.A., Bagchi, R., 2007. Phosphorus supply influences heathland responses to atmospheric nitrogen deposition. Environ. Pollut. 148, 191-200. https://doi.org/10. 1016/j.envpol.2006.10.034

Pinheiro, J., Bates, D., DebRoy, S., Sarkar, D., R Core Team, 2016. nlme: Linear and Nonlinear Mixed Effects Models. R Package Version. 31-128 http:/CRAN. R-project.org/package $=$ nlme.

Power, S.A., Green, E.R., Barker, C.G., Bell, J.N.B., Ashmore, M.R., 2006. Ecosystem recovery: heathland response to a reduction in nitrogen deposition. Glob. Chang. Biol. 12, 1241-1252. https://doi.org/10.1111/j.1365-2486.2006.01161.x.

Prins, A.H., Berdowski, J.J.M., Latuhihin, M.J., 1991. Effect of $\mathrm{NH}_{4}$-fertilization on the maintenance of a Calluna vulgaris vegetation. Plant Biol. 40, 269-279. https:// doi.org/10.1111/j.1438-8677.1991.tb01558.x.

R Core Team, 2016. R: a Language and Environment for Statistical Computing. R Foundation for Statistical Computing, Vienna, Austria https://www.R-project.org/.

Reich, P.B., 2009. Elevated $\mathrm{CO}_{2}$ reduces losses of plant diversity caused by nitrogen deposition. Science 326, 1399-1402. https://doi.org/10.1126/science.1178820.

Rivero Fernández, C., Rabago Juan-Aracil, I., Sousa Carrera, M., Lorente Ibáñez, M. Schmid, T., 1996. Cálculo y cartografía de cargas críticas para España. Aplicación del modelo SMB, CIEMAT, Madrid.

Rose, R.J., Webb, N.R., Clarke, R.T., Traynor, C.H., 2000. Changes on the heathlands in Dorset, England, between 1987 and 1996. Biol. Conserv. 93, 117-125. https:// doi.org/10.1016/S0006-3207(99)00047-6.

Sagarin, R.D., Gaines, S.D., Gaylord, B., 2006. Moving beyond assumptions to understand abundance distributions across the ranges of species. Trends Ecol. Evol. 21, 524-530. https://doi.org/10.1016/j.tree.2006.06.008.

Soons, M.B., Hefting, M.M., Dorland, E., Lamers, L.P.M., Versteeg, C., Bobbink, R., 2017. Nitrogen effects on plant species richness in herbaceous communities are more widespread and stronger than those of phosphorus. Biol. Conserv. 212, 390-397. https://doi.org/10.1016/j.biocon.2016.12.006.

Southon, G.E., Green, E.R., Jones, A.G., Barker, C.G., Power, S.A., 2012. Long-term nitrogen additions increase likelihood of climate stress and affect recovery from wildfire in a lowland heath. Glob. Chang. Biol. 18, 2824-2837. https://doi.org/10. 1111/j.1365-2486.2012.02732.x.

Southon, G.E., Field, C., Caporn, S.J.M., Britton, A.J., Power, S.A., 2013. Nitrogen deposition reduces plant diversity and alters ecosystem functioning: field-scale evidence from a nationwide survey of UK heathlands. PLoS One 8 (4), e59031https: //doi.org/10.1371/journal.pone.0059031

Stevens, C.J., Payne, R.J., Kimberley, A., Smart, S.M., 2016. How will the semi-natural vegetation of the UK have changed by 2030 given likely changes in nitrogen deposition?. Environ. Pollut. 208, 879-889. https://doi.org/10.1016/j.envpol.2015. 09.013 .

Taboada, A., Marcos, E., Calvo, L., 2016. Disruption of trophic interactions involving the heather beetle by atmospheric nitrogen deposition. Environ. Pollut. 218, 436-445. https://doi.org/10.1016/j.envpol.2016.07.023. 
Valbuena, L., Tárrega, R., Luis-Calabuig, E., 2000. Seed banks of Erica australis and Calluna vulgaris in a heathland subjected to experimental fire. J. Veg. Sci. 11 161-166. https://doi.org/10.2307/3236795.

Van den Berg, L.J.L., Peters, C.J.H., Ashmore, M.R., Roelofs, J.G.M., 2008. Reduced nitrogen has a greater effect than oxidised nitrogen on dry heathland vegetation. Environ. Pollut. 154, 359-369. https://doi.org/10.1016/j.envpol.2007.11.027.

Van den Berg, L.J.L., Vergeer, P., Richs, T.C.G., Smart, S.M., Guest, D., Ashmore, M.R., 2011. Direct and indirect effects of nitrogen deposition on species composition change in calcareous grasslands. Glob. Chang. Biol. 17, 1871-1883. https:// doi.org/10.1111/j.1365-2486.2010.02345.x.

Velle, L.G., Nilsen, L.S., Vandvik, V., 2012. The age of Calluna stands moderates post-fire regeneration rate and trends in northern Calluna heathlands. Appl. Veg. Sci. 15, 119-128. https://doi.org/10.1111/j.1654-109X.2011.01144.x.

Venables, W.N., Ripley, B.D., 2002. Modern Applied Statistics with S, fourth edition Springer, New York.
Viejo, R.M., Martínez, B., Arrontes, J., Astudillo, C., Hernández, L., 2011. Reproductive patterns in central and marginal populations of a large Brown seaweed: drastic changes at the southern range limit. Ecography 34, 75-84. https://doi.org/10.1111/ j.1600-0587.2010.06365.x.

Von Oheimb, G., Härdtle, W., Falk, K., Gerke, A.-K., Meyer, H., Drees, C., Matern, A., 2009. Is Calluna vulgaris a suitable bio-monitor of management-mediated nutrient pools in heathland ecosystems?. Ecol. Indic. 9, 1049-1055. https://doi.org/ 10.1016/j.ecolind.2008.12.005.

Von Oheimb, G., Power, S.A., Falk, K., Friedrich, U., Mohamed, A., Krug, A., Boschatzke, N., Härdtle, W., 2010. N:P ratio and the nature of nutrient limitation in Calluna-dominated heathlands. Ecosystems 11, 317-327. https://doi.org/10. 1007/s10021-010-9320-y.

Webb, N.R., 1998. The traditional management of European heathlands. J. Appl. Ecol. 35, 987-990. https://doi.org/10.1111/j.1365-2664.1998.tb00020.x. 